\title{
The not so identical twins: (dis)similarities between reactive electrophile and oxidant sensing and signaling
}

\author{
Marcus J. C. Long ${ }^{\ddagger}$ Kuan-Ting Huang†, and Yimon Aye+* \\ +Swiss Federal Institute of Technology in Lausanne (EPFL), 1015 Lausanne, Switzerland \\ ‡University of Lausanne, 1015 Lausanne, Switzerland \\ ${ }^{*}$ Correspondence: yimon.aye@epfl.ch
}

Abstract

In this tutorial review, we compare and contrast the chemical mechanisms of electrophile/oxidant sensing, and the molecular mechanisms of signal propagation. We critically analyze biological systems in which these different pathways are believed to be manifest and what the data really mean. Finally, we discuss applications of this knowledge to disease treatment and drug development.

Keywords: RES; ROS; reactive chemical species; electrophile signaling; cysteine; LDE; T-REX; G-REX; Chemical Biology 


\section{Key learning points}

- Despite being promiscuous, reactive electrophilic and oxidative species (RES and ROS) show nuanced biological properties and functions driven by context-dependent covalent modification of specific proteins.

- Different RES and ROS chemotypes have different interaction preferences, due to different intrinsic rates of reaction with specific functional groups. Molecules with lower intrinsic rates of reaction are most likely to be used selectively by biology.

- Modulation of biological processes triggered as a result of specific interactions between specific RES/ROS and specific proteins, occurs particularly with relatively low-reactivity RES/ROS. Proteins involved in these processes typically manifest elevated reaction rates with (often specific) RES/ROS.

- Methods to detect both free RES/ROS and RES/ROS-derived modifications on proteins can give key insight into localized elevation of reactive signals, but are limited in informing RES/ROS-signaling functions and consequences of such modifications.

- New methods to probe on-target signaling can complement the above methods in examining the role of protein- as well as RES/ROS-chemotype-specific modifications in biological signaling. 
Biological systems generate a plethora of chemically-diverse reactive species, which can be formed in metabolic processes, and during stress or cell death. The environment is also a source of such molecules, including through inhalation of smoke and in our diet. Unsurprisingly, reactive molecules were once considered unwanted biological byproducts and imperfections, and/or negative consequences of lifestyle and the oxidizing environment we live in. Certainly, since reactive molecules display broad reactivity with numerous chemical groups important in protein and DNA structure and function (e.g., thiol, amine, aromatic motifs), reactive molecules are inherently promiscuous and have the proclivity to interact pleiotropically with a wide range of biological macromolecules. Such chemical rabble-rousers may be expected to be detrimental to cells. However, emerging data show that many reactive biological molecules can impact or rewire biological signaling pathways meaningfully and specifically. This finding has spurred new ways of experimental planning and performing experiments, as well as new technologies and translational applications of reactive species. Such studies required innovative deployment of chemical knowledge and understanding in complex biological systems. This is because reactive molecules cannot inherently manifest the selectivity of enzymological proteinmodification processes (e.g., phosphorylation or ubiquitination) that proceed by amplifying rates of chemical reactions that do not occur spontaneously; nor are reactive species as amenable to study by traditional genetic methods.

Reactive biological molecules are often clumped together as "reactive species", "oxidative stressors", or "redox stressors". However, not all reactive species, e.g., reactive electrophile species (RES), are oxidants: thus, sub-categorizing reactive species as RES and reactive oxygen species (ROS), can be helpful, although both sub-classes belie the rich chemistry and biology undertaken by the thousands of biologically-relevant small molecules that fall under each class. This review seeks to provide a framework to understand reactive signals, and to further interrogate their functions/behavior. We will deal with a select number of oxidants and electrophiles as exemplars; we do not intend to cover all chemical space or eventualities. Rather, we set out to exemplify the chemical, biochemical, and 
biological concepts needed to critically evaluate the literature, propose new ideas, and design meaningful experiments with which to put those ideas to the test.

We commence with basic chemical properties of common oxidants and electrophiles. These properties define intrinsic behaviors of reactive molecules, such as reactivity/chemoselectivity, and stability of products. We will then move forward with a short discussion on how reactive species are generated in biological systems. Appreciation of the processes involved in the genesis of reactive entities is crucial, as their origins are key determinants of their interactomes. This is because reactive molecules cannot travel the length of the cell ${ }^{1}$ and so must act locally. We will compare and contrast, e.g., the transition states of reaction of different RES/ROS with proteins; such considerations have important ramifications for understanding reactive residues and their substrates. We will also compare and contrast reaction products, in terms of stability and signal transmissibility. All these properties define our ability to detect and measure ROS/RES concentrations. Finally, we will discuss how once target labeling has occurred, signaling can occur.

In this piece, we will use rates of reaction of representative RES/ROS with prototypical nucleophiles/reductants in buffered aqueous environments approximating what is believed to occur in a normal cell. These numbers are useful for budding aficionados, and have general relevance to inhibitor design and other aspects of biology. This is because inherent reaction rates can be used as a yardstick to decide if a proposed process be feasible, and to rank general reactivities of different reagents under similar conditions. Representative rates can also help assess whether a specific contribution to an overall observed rate, such as deprotonation of a thiol, be sufficient to account for an observed rate, for instance between a protein thiol and an electrophile of interest. However, it is crucial not to overextend the analogy: subtle structural/environmental changes that may even occur in simple chemical systems can have extreme consequences on reactivity profiles, including regioselectivity and stereoselectivity. Examples can be derived from the 24 -fold difference in rates between reactivities of carbonyls in cyclohexanone versus cyclopentanone. This rate difference is a 
common feature of exocyclic double bonds in 5- and 6-membered rings, which is inverted for endocyclic double bonds ${ }^{2}$. Furthermore, once a protein is involved in a chemical process, the chemistry that occurs in buffered aqueous solution may not be representative, oftentimes even on a relative scale. Such changes can be ascribed to solvation/desolvation and changes in, e.g., dielectric constants/positioning of specific charges, that can occur due to the unique environment offered by proteins. To give an example of how solvation and local polarization is important: in the gas phase tert-butanol is significantly more acidic than methanol, whereas the opposite trend, commonly reported in textbooks, is observed in water. Thus, we implore readers not to be lured into blanketly assuming that simple in vitro-derived rate constants or experiments, even on "representative proteins", can be extrapolated to, or within, complex systems.

\section{Reactive chemical species}

ROS discussed are hydrogen peroxide $\left(\mathrm{H}_{2} \mathrm{O}_{2}\right)$, oxygen radicals, superoxide, and singlet oxygen $\left({ }^{1} \mathrm{O}_{2}\right)(\mathrm{Fig}$. S1A). RES discussed are 4-hydroxynonenal (HNE, a representative lipid-derived RES), and fumarate (an essential endogenous metabolite, whose core structure is present in several approved drugs $^{3,4}$ ) (Fig. S1B).

ROS

Peroxides. The biological oxidants, $\mathrm{H}_{2} \mathrm{O}_{2}$, a large number of structurally/chemically similar peroxides species, and also other isolobal oxidants, e.g., $\mathrm{HOCl}$, share a core defining feature: an oxygenheteroatom bond, which is weak due to electron-electron repulsion and short length. These molecules consequentially possess a low-lying lowest unoccupied molecular orbital (LUMO), the $\sigma^{*}$ of the Oheteroatom single bond. This species is particularly prone to nucleophilic attack by good nucleophiles (bearing a high energy highest occupied molecular orbital, HOMO). Thus, hydrophobic aliphatic aminoacid sidechains and alcohol-containing sidechains do not react appreciably with peroxides. Aromatic sidechains do not react with peroxides, but react with more reactive isolobal oxidants, e.g., $\mathrm{HOCl}$ (Fig. 1A: left). The most common biological nucleophiles that engage with peroxides are thiols, which in 


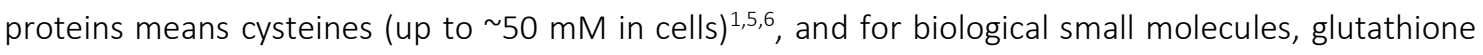
(around $5 \mathrm{mM}$ in cells) $)^{1}$ (Fig. S1C). For a typical thiol, the second order rate constant $\left(k_{2}\right)$ with peroxide is $\sim 1-2 \mathrm{M}^{-1} \mathrm{~s}^{-1}$ at neutral $\mathrm{pH}$. Complete deprotonation of the thiol increases this rate to only $\sim 20 \mathrm{M}^{-1} \mathrm{~s}^{-1}$. This rate is underwhelming. For $1 \mu \mathrm{M} \mathrm{H}_{2} \mathrm{O}_{2}$ (which is up to $500 x$ cellular basal levels ${ }^{7}$, requiring $\sim 400$ $\mu \mathrm{M}$ external $\mathrm{H}_{2} \mathrm{O}_{2}$ to be achieved intracellularly), and $1 \mu \mathrm{M}$ protein thiol, the $2^{\text {nd }}$ order rate constant $k_{2}$ is $20 \mathrm{M}^{-1} \mathrm{~s}^{-1}$. The time required for initial $\mathrm{H}_{2} \mathrm{O}_{2}$ concentration to deplete by half (first $t_{1 / 2}$ ) is $\sim 14 \mathrm{~h}$ (Fig. 1B: top panel). Lowering protein-thiol concentration leads to a pseudo-first order $t_{1 / 2} \sim 10 \mathrm{~h}$ (Fig. 1B: middle panel). However, peroxiredoxin can reduce $\mathrm{H}_{2} \mathrm{O}_{2}$ rapidly at close to diffusion control, reducing $\mathrm{H}_{2} \mathrm{O}_{2}$ 's cellular $t_{1 / 2}$ to $<1 \mathrm{~s}$. Thus, typical cysteine thiolates cannot intrinsically achieve meaningful occupancy against bulk cellular processes. Elevated $\mathrm{H}_{2} \mathrm{O}_{2}$ concentrations can mitigate these concerns, although even at $10 \mu \mathrm{M}$ intracellular $\mathrm{H}_{2} \mathrm{O}_{2}$, pseudo-first order oxidation $t_{1 / 2}$ is, $\sim 1 \mathrm{~h}$ (Fig. 1B: lowest panel), again ignoring clearance. Depletion of subcellular defense mechanisms may promote direct protein labeling. Although such effects do not improve direct protein labeling kinetics, they could severely compromise cells, leading to artifactual results.

The ability of peroxiredoxin to achieve such a rapid rate of reaction with $\mathrm{H}_{2} \mathrm{O}_{2}$ shows that mechanisms beyond deprotonation can promote thiol reactivity by orders of magnitude more than deprotonation. For instance, $\mathrm{HOCl}$ and $\mathrm{HOBr}$, oxidize thiols with $k_{2} \sim 10^{8}-10^{7} \mathrm{M}^{-1} \mathrm{~s}^{-1}$. This $\sim 10^{7}$-fold rate acceleration over the equivalent reaction with $\mathrm{H}_{2} \mathrm{O}_{2}$, occurs ostensibly by stabilizing the leaving group and/or polarizing the O-heteroatom bond (lowering the $\left.\sigma^{*}\right)$ (Fig. 1A: right), and certainly does not affect the HOMO of the nucleophile. Conversely, lowering the pKa of a thiol, actually reduces the potency of the thiolate nucleophile for several biological oxidants, although not all ${ }^{1}$. This is because reducing pKa lowers the thiolate $\mathrm{HOMO}^{1}$ (Fig. 1C). Thus, tuning the thiol pKa, in the absence of other aspects that elevate intrinsic thiol nucleophilicity, will not alter the above arguments. Some biological oxidants also appear to react in an acid-catalyzed manner ${ }^{8}$. 
A

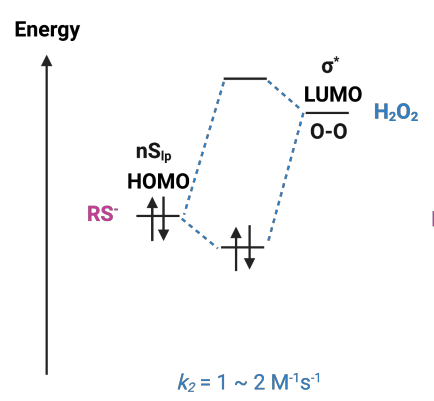

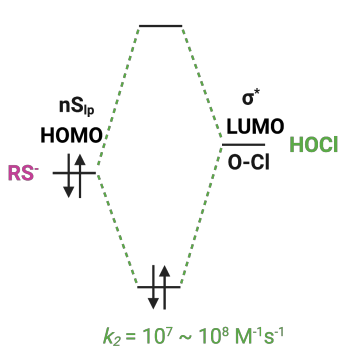

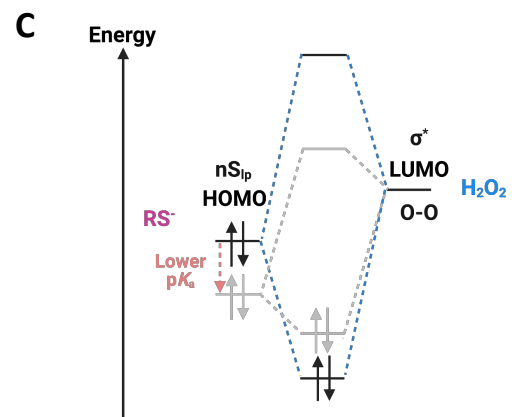

B

Reaction rate $=k\left[\mathrm{H}_{2} \mathrm{O}_{2}\right][\mathrm{RS}], k=20 \mathrm{M}^{-1} \mathrm{~s}^{-1}$

$$
\begin{aligned}
& \text { If }\left[\mathrm{H}_{2} \mathrm{O}_{2}\right]_{0}=[\mathrm{RS}]_{0}=1 \mu \mathrm{M} \\
& \rightarrow t_{1 / 2}(1)=1 / k\left[\mathrm{H}_{2} \mathrm{O}_{2}\right]_{0} \sim 14 \mathrm{~h} \\
& \text { (Second order reaction) }
\end{aligned}
$$

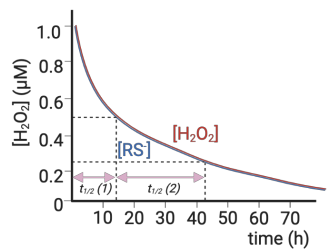

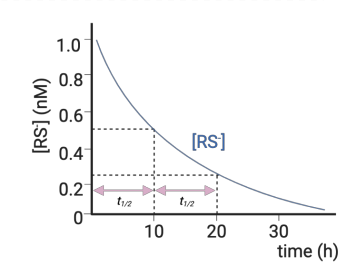
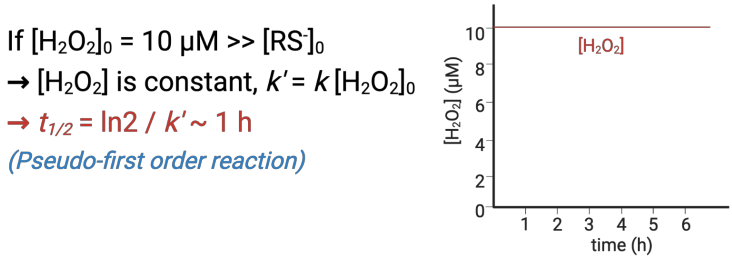

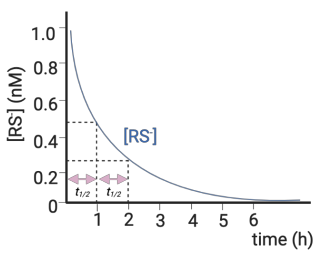

Figure 1. (A) Peroxides and some isolobal oxidants, e.g., $\mathrm{HOCl}$, have a lower LUMO (the $\sigma^{*}$ of the $\mathrm{O}-\mathrm{O}$ or $\mathrm{O}-\mathrm{X}$, where $X$ is heteroatom), and can be attacked by nucleophiles. As the LUMO of $\mathrm{HOCl}$ is lower than $\mathrm{H}_{2} \mathrm{O}_{2}$, the reaction rate of $\mathrm{HOCl}$ with nucleophiles is higher than that of $\mathrm{H}_{2} \mathrm{O}_{2}$. $\mathrm{HOCl}$ oxidizes thiols with $k_{2}=10^{7}-10^{8} \mathrm{M}^{-1} \mathrm{~s}^{-1}$; $\mathrm{H}_{2} \mathrm{O}_{2}$ oxidizes thiols with $k_{2}=1-2 \mathrm{M}^{-1} \mathrm{~s}^{-1}$. $\mathrm{nS}$ ip: non-bonding lone pairs on sulfur. (B) When initial concentrations of $\mathrm{H}_{2} \mathrm{O}_{2}$ and $\mathrm{RS}^{-}$(i.e., $\left[\mathrm{H}_{2} \mathrm{O}_{2}\right]_{0}$ and $\left[\mathrm{RS}^{-}\right]_{0}$ ) are equal, the rate of reaction is determined by $\left[\mathrm{H}_{2} \mathrm{O}_{2}\right]$ and $\left[\mathrm{RS}^{-}\right]$, and cellular half-life of $\mathrm{H}_{2} \mathrm{O}_{2}\left(t_{1 / 2}\right)$, changes with conversion (top panel). However, when $\left[\mathrm{H}_{2} \mathrm{O}_{2}\right]_{0}$ is much higher than $\left[\mathrm{RS}^{-}\right]_{0}$, the reaction becomes pseudo first-order, the half-life no longer changes with conversion. The rate of reaction depends on $\left[\mathrm{H}_{2} \mathrm{O}_{2}\right]$, but this is invariant across the reaction (middle and bottom panels). The plots show timedependent changes of $\left[\mathrm{H}_{2} \mathrm{O}_{2}\right]$ and $\left[\mathrm{RS}^{-}\right]$, with the first and second half-lives of [RS-] indicated. (C) Nucleophiles, such as thiol, can attack peroxides. Lowering $\mathrm{p} K_{\mathrm{a}}$ of a thiol lowers the HOMO of the corresponding thiolate, resulting in a smaller orbital overlap between thiolate and electrophile, and a slower reaction rate. $\mathrm{nS}$ ip: nonbonding lone pairs on sulfur. 
Selenium-containing residues can also attack $\mathrm{H}_{2} \mathrm{O}_{2}$. As selenium is a lower period element than cysteine (i.e., its nucleus is more shielded, and electrons are further away from the nucleus), the reaction of selenocysteine with peroxides is significantly faster than the reaction with thiol-based nucleophiles (Fig. 2), although the difference in rate constants is $\mathrm{pH}$-dependent: between selenocysteine and cysteine anions there is a $\sim 15$-fold difference in rate constant ( $\mathrm{RSe}^{-}$and $\mathrm{H}_{2} \mathrm{O}_{2}$ both at $1 \mu \mathrm{M}$ will react with a first $t_{1 / 2}=42$ minutes). At neutral $\mathrm{pH}$, the proportion of the anion in selenocysteine is much higher than cysteine $(p K a$, selenocysteine $=4.8 ; p K a$, cysteine $=8.5$; a consequence of the weak Se-H bond and the stability of $\mathrm{Se}^{-}$conjugate base), rendering the apparent differences in $k_{2} \sim 400$-fold ${ }^{9}$. These rate increases are sizeable, but neither the magnitude of increase, nor the absolute $k_{2}$ values, are large compared to enzyme-mediated rate enhancement. Indeed, the appearance of selenocysteine in enzymes may not be to promote oxidation rates, but rather to promote resistance to overoxidation ${ }^{9}$. Critically, the selenocysteome remains an area of active research, and it is possible that numerous selenocysteines in proteins are undiscovered.

Methionine oxidation (Fig. 2) has been proposed to be a biologically-relevant modification, for instance, in prion diseases ${ }^{10}$. Methionine oxidation by peroxides has $k_{2} \sim 0.01 \mathrm{M}^{-1} \mathrm{~s}^{-1}$, two orders of magnitude slower than cysteine. However, in low pH environments, and particularly in the presence of oxidants that react with cysteine at close to diffusion control, cysteine and methionine show similar reactivity. Methionine may indeed be more nucleophilic than cysteine with $\mathrm{HOCl}\left(\sim 10^{8} \mathrm{M}^{-1} \mathrm{~s}^{-1}\right)^{11}$, and for $\mathrm{HOBr}$, there are similar rates for the twain $\left(\sim 10^{7} \mathrm{M}^{-1} \mathrm{~S}^{-1}\right)^{11}$. Peracids (Fig. S1D), which are more oxidizing than peroxides (due to a lower $\sigma^{*}$ /better leaving group), can oxidize methionine with $k_{2} \sim 1-5 \mathrm{M}^{-1} \mathrm{~s}^{-1}{ }^{12}$, which based on the above discussion, is unlikely to be particularly relevant. Cysteine reacts several orders of magnitude faster with peracids than methionine ${ }^{13}$.

Although nitrogen oxidation by peroxide is common industrially, tryptophan, histidine, and lysine oxidation by peroxide are not commonly observed and likely occur significantly slower than for (seleno)cysteine (Fig. 2). For instance, histidine oxidation by peracetic acid has $k_{2} \sim 1 \mathrm{M}^{-1} \mathrm{~s}^{-1}{ }^{14}$. Oxidation 
of these residues is likely to be irreversible. $\mathrm{HOCl}$ and $\mathrm{HOBr}$ can react with tryptophan, histidine and lysine, and the $\alpha$-amino group, although these rates are at least several folds slower than for the two sulfur-containing amino acids ${ }^{11}$. Indeed, $\mathrm{HOCl}$ and $\mathrm{HOBr}$ have the power to create novel functional groups, which may offer new modes of regulation. Oxidation of lysine can lead to formation of lysine nitrile $^{15}$ (Fig. 2). Enzymatic oxidation of lysine by myeloperoxidase/peroxide/chloride is known, leading to adipic acid derivatives (Fig. 2). Halogenation of amino acids is also possible ${ }^{16}$. Such modifications, particularly on long-lived proteins could therefore be relevant, especially to aging, or in cells possessing high oxidative power. However, this aspect has not been particularly thoroughly investigated.

Reduction of the product (seleno)cysteine sulfenic acids (Fig. 2) is rapid in the presence of smallmolecule reductants, like glutathione and enzymatic reductants. Reduction often proceeds through a disulfide or selenosulfide that are rapidly reduced further. However, when formed on a protein sulfenic acids, dithiols, or other similar oxidation states of sulfur, may be stable and many of these intermediates have signaling properties. For methionine sulfoxide re-reduction requires enzymatic catalysis, making methionine redox regulation overall more context dependent ${ }^{1}$. Given that the production and reduction of methionine sulfoxide is very locale dependent, it is possible that there is a rich locallycontrolled oxidation chemistry of this residue. However, this is likely either a slow response system, or mediated by oxidants that occur in specific cell types. 

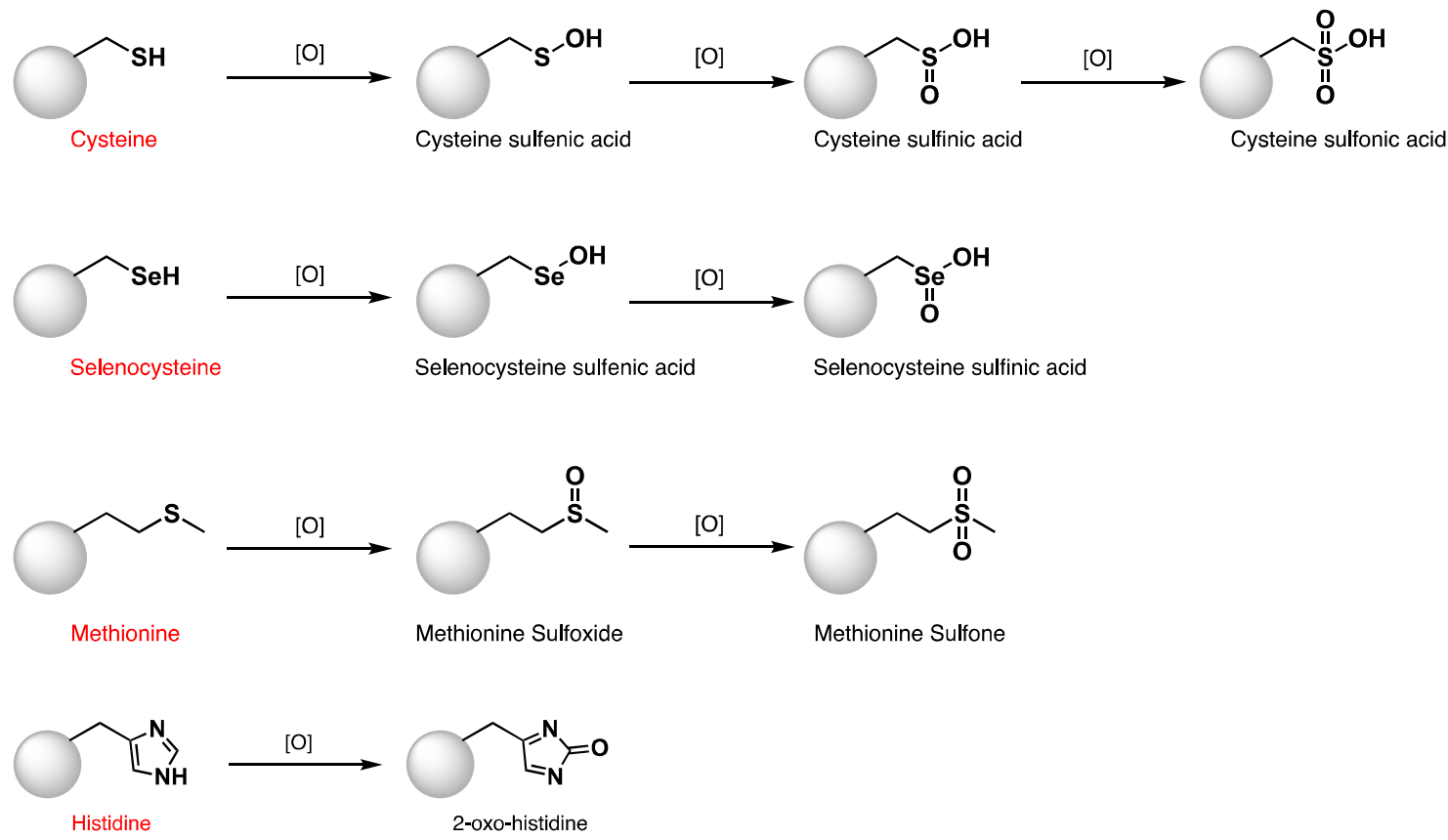

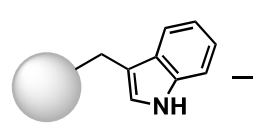

Tryptophan

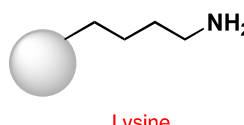

Lysine

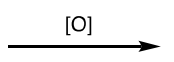

$\mathrm{PO}^{2}$

$\downarrow$

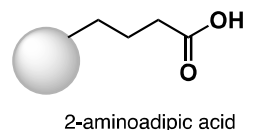

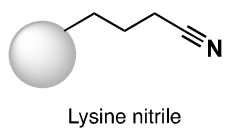

Lysine nitrile

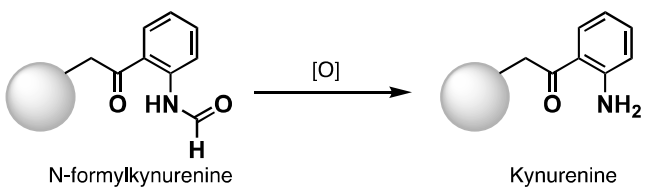

Figure 2. A selection of oxidation process of amino acid side chains by ROS. 


\section{Alkoxy/peroxy-radicals}

These are an equally diverse series of reagents to the peroxides. These radicals are significantly more reactive than peroxide, and have consequentially larger substrate repertoires. There are two principal flavors of neutral oxygen radicals formed in biology: alkoxy- and peroxy-radicals (Fig. 3A). Alkoxyradicals are significantly more reactive than peroxy-radicals, where electrons on the geminal oxygen atom can delocalize into the electron-deficient oxygen radical, offering stabilization. Owing to their higher stability, peroxy-radicals are generally more regio/chemoselective than alkoxy-radicals, and are usually considered to only target cysteine, methionine, and aromatic side chains. Alkoxy-radicals can oxidize most amino-acid sidechains (Fig. 3A). Nevertheless, as electron-deficient, or electrophilic, radicals, both favor abstraction from weak bonds and at positions that would form stabilized carbocations (captodative stabilization, and high substitution) ${ }^{1}$. Abstraction at the $\alpha$-hydrogen of the amino acid (which forms a tertiary captodative stabilized radical, in all cases bar glycine) thus is favored, and is also one of the most destructive events (Fig. 3B). It does, however, seem that the rate of this process is lower than may be expected de novo ${ }^{1}$. Carbon radical products in general can trap out molecular oxygen, forming an intermediate peroxide radical that can abstract another hydrogen atom, forming another radical, and a peroxide. If the latter be geminal to a heteroatom, this can liberate peroxide, and lead to a carbonyl/imine. Should the initial abstraction have occurred at the $\alpha$-proton of an amino acid, this causes fragmentation of the peptide backbone, explaining the danger of $\alpha$-oxidation of peptides. Organic peroxides can also undergo 1,2-migration and elimination among others. Formation of thiyl radicals, may be reversible as this can lead to (per)sulfenic acids. For other modes of attack, these species enact irreversible chemistry. The only means to undo such effects is protein degradation. 
A

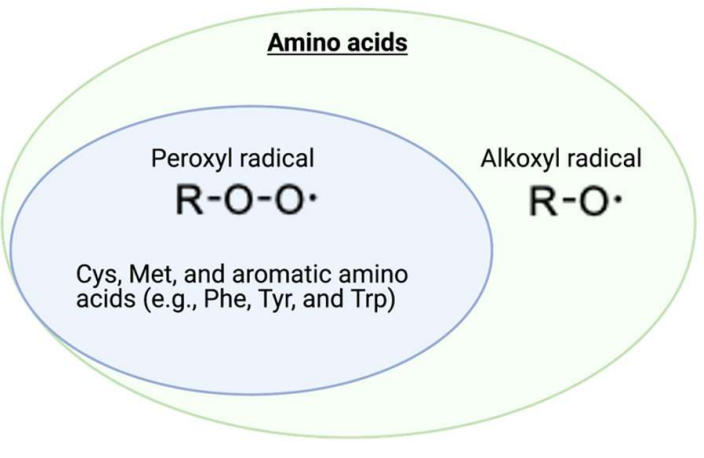

B
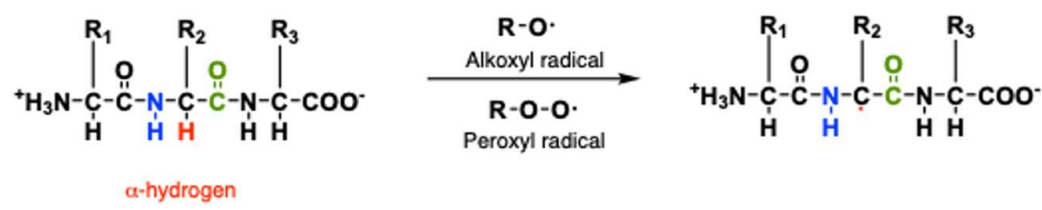

Figure 3. (A) Alkoxyl radicals are generally more reactive than peroxyl radicals. The latter is considered to only target cysteine, methionine, and amino acids with aromatic side chains. (B) Alkoxyl and peroxyl radicals are more reactive than peroxides, and can oxidize peptides or proteins. These radicals favor removing activated $\mathrm{C}-\mathrm{H}$ bonds, e.g., the $\alpha$-hydrogen (colored red), instead of strong bonds like $\mathrm{N}-\mathrm{H}$ and deactivated $\mathrm{C}-\mathrm{H}$, e.g., to form primary radicals.

\section{Superoxide and ${ }^{1} \mathrm{O}_{2}$}

Our next two oxidants are unique, in the sense that they are each the only molecules in their specific classes. Both are forms of molecular oxygen: superoxide is the product of one electron reduction; singlet oxygen $\left({ }^{1} \mathrm{O}_{2}\right)$ is an electronically excited form of triplet oxygen. ${ }^{1} \mathrm{O}_{2}$ behaves as an oxygen molecule bearing a formal double bond, as opposed to having diradical character. ${ }^{1} \mathrm{O}_{2}$ reacts rapidly with cysteine, methionine and aromatic amino acids, but can also react with unsaturated lipids through Alder-ene or Diels Alder-type chemistry. Lipid oxidation by ${ }^{1} \mathrm{O}_{2}$ affords peroxides, which display reactivity, akin to that discussed above. Superoxide is the only member of the principal oxidants we discuss that is charged at neutral $\mathrm{pH}$. This factor endows superoxide with a relatively unusual diffusivity and chemoselectivity: superoxide is likely unable to diffuse across membranes as effectively as neutral oxidants; and although superoxide reacts with cysteine to create a thiol radical (rate constants $\sim 10^{5} \mathrm{M}^{-}$ $\left.{ }^{1} \mathrm{~s}^{-1}\right)^{17}$ many folds faster than peroxide with thiols, superoxide has a preference for other biomolecules such as heme/iron metal centers, which it can oxidize with rates $>10^{6} \mathrm{M}^{-1} \mathrm{~s}^{-1}{ }^{18}$. Superoxide also reacts 
particularly rapidly with aromatic sidechain radicals, such as tryptophan or tyrosine radicals $\left(10^{9} \mathrm{M}^{-1} \mathrm{~s}^{-}\right.$ $\left.{ }^{1}\right)^{17}$. Intriguingly, superoxide only interacts with methionine with a rate constant around $1 \mathrm{M}^{-1} \mathrm{~s}^{-1} \quad 17,18$. Thus, despite being less cysteine-centric in its reactivity than peroxide, superoxide is considerably more chemoselective for cysteine versus methionine. It is noteworthy, finally to state that superoxide has a pKa around 5. The product of protonation of superoxide, is the peroxyl radical. This has a much broader reaction spectrum, and is more membrane diffusive. Again, thiyl radical formation may be reversible, although the rest of these events, are likely irreversible.

RES

The interaction of electrophiles with proteins and its link to disease has been known since the 1950's, due to work from Betty and Jim Millar ${ }^{19}$. Although a generalization, $\alpha, \beta$-unsaturated carbonyl species overall have reactivities with biological nucleophiles similar to peroxide. The best studied $\alpha, \beta-$ unsaturated electrophile, 4-hydroxynonenal (HNE) (Fig. S1), shows an almost exclusive preference to react with soft nucleophiles, i.e., cysteine with $k_{2} \sim 1 \mathrm{M}^{-1} \mathrm{~s}^{-1}$ at neutral $\mathrm{pH} . \mathrm{k}_{2}$ rises to $\sim 20 \mathrm{M}^{-1} \mathrm{~s}^{-1}$ for the corresponding thiolate ${ }^{1}$. Similar to what we saw with peroxide versus $\mathrm{HOCl}$, increasing the electron deficiency of the electrophile elevates $k_{2}$ : which for 4-oxononenal (ONE) (Fig. S1) is $150 \mathrm{M}^{-1} \mathrm{~s}^{-1}$ at neutral $\mathrm{pH}$. Contributions here arise from increasing the coefficient of positive charge at the $\beta$-carbon, lowering of the LUMO, both through induction, and also reduction of steric effects at the $\beta$-carbon (Fig. 4A). Intriguingly, there are reports that ONE shows higher chemoselectivity for cysteine and histidine over Iysine than $\mathrm{HNE}^{20}$, further highlighting that modest changes in functionality should not be taken lightly. ONE brings in a new aspect of complexity, regioselectivity, since both olefinic carbons may be sites of nucleophilic attack. One would expect that addition $\beta$-to the aldehyde would dominate over addition $\beta$-to the ketone (as the enolate intermediate is more stable in the former). It is possible that assessing the regioselectivity of such bifunctional compounds could aid understanding of how proteins react with enals/enones. However, in reality, such precision assessments of chemical regioselectivity are hard to perform and there are no such reports we know of. Intriguingly, the change in $k_{2}$ going from HNE to 
ONE is small relative to the change from peroxide to $\mathrm{HOCl}$. This difference can be ascribed to the fact that it is difficult to achieve the same amount of electron withdrawing power/anion stabilization in the conjugate base of an $\alpha, \beta$-unsaturated electrophile as is possible with, for instance, $\mathrm{HOCl}$. So, the fold changes in terms of electron-withdrawing capacity are to some extent built into each system and retain rates relatively low for most biological electrophiles. Further highlighting the importance of steric factors: acrylaldehyde shows a $k_{2} \sim 500 \mathrm{M}^{-1} \mathrm{~s}^{-1}$ at neutral $\mathrm{pH}^{21}, 500$ times more than HNE (above). $k_{2}$ drops to $0.2 \mathrm{M}^{-1} \mathrm{~s}^{-1}$ for acrylamide ${ }^{22}$, demonstrating the importance of electron-withdrawing nature/stability of the enolate intermediate in promoting activity. The ultimate conclusion here is that within specific classes of biological electrophiles, there is a large scope for improvements in rates that does not exist for many ROS (especially those that react inherently rapidly). Indeed, the acrylamide function is the core reactive component of several approved electrophilic drugs, many of which show $k_{2}>10^{5} \mathrm{M}^{-1} \mathrm{~s}^{-1}$ for conjugate addition ${ }^{1,23}$. We will show examples of similar behavior on endogenous electrophiles with matched proteins later.

Unlike peroxide, products of selenocysteine conjugate addition to electrophiles are not commonly considered, although these have been reported ${ }^{24}$. The lack of examples may be attributable to $\beta$ selenylcarbonyls being prone to elimination of selenide or "retro-ing" either directly, or during analysis (Fig. 4B). Indeed, it is probable that limitations in detection methods have restricted our ability to observe specific modifications of several residues. For instance, $\beta$-mercapto-nitro species, whose $\alpha$ protons are more acidic than $\beta$-mercapto-aldehydes, are prone to retro-ing ${ }^{1}$ (Fig. $4 \mathrm{~B}$ : top and middle rows). As $\beta$-seleno-carbonyls are likely more acidic than $\beta$-mercapto-carbonyls, and selenide is a better leaving group than sulfide, it is likely that $\mathrm{E} 1_{\mathrm{CB}}$-type retro-ing ${ }^{25}$ (a process for which deprotonation and elimination are often co-rate-limiting) for selenocysteine adducts, is common (Fig. 4B: bottom row). However, selenium has an overall richer chemistry than sulfur, so there are other possibilities: $\beta$-seleno alcohols, like the product of selenocysteine adduction to HNE, can form olefins ${ }^{26}$, similarly to $\beta$-silyl alcohols. This process is accelerated at low pH, conditions often deployed to favor identification of electrophile-reactive selenocysteines, by analogy to experiments profiling selenocysteine oxidation. 
Low $\mathrm{pH}$ conditions are used for elution in many immunoprecipitation strategies, and in the majority of (although not all) gel stains. Thus, context-specific chemotype arrangements could also be important in determining stabilities of these intermediates, and each processing step needs to be considered carefully when planning experiments aiming to identify a specific modification.

There is also evidence that $\alpha, \beta$-unsaturated carbonyl species, e.g., HNE, interact with histidine $\left(k_{2}\right.$ $\left.\sim 0.002 \mathrm{M}^{-1} \mathrm{~s}^{-1}\right)$ and lysine $\left(k_{2} \sim 0.001 \mathrm{M}^{-1} \mathrm{~s}^{-1}\right.$; although similar products are often unstable). Indeed, rapid labeling of histidine by some electrophiles $\left(10^{6} \text {-fold above background }\right)^{27}$ occurs in serine proteases. It has been reported that histidine-adducts may dominate over cysteine adduction in biological systems ${ }^{1}$, although this may only occur at high electrophile concentrations or after prolonged exposure periods. This result is indicative of compromised redox regulation in cells favoring histidine adduction. However, since the majority of reports show cysteine is significantly more reactive than histidine and numerous biologically-relevant cysteine modifications have been reported, cysteine is likely the major protagonist in electrophile signaling. 
A

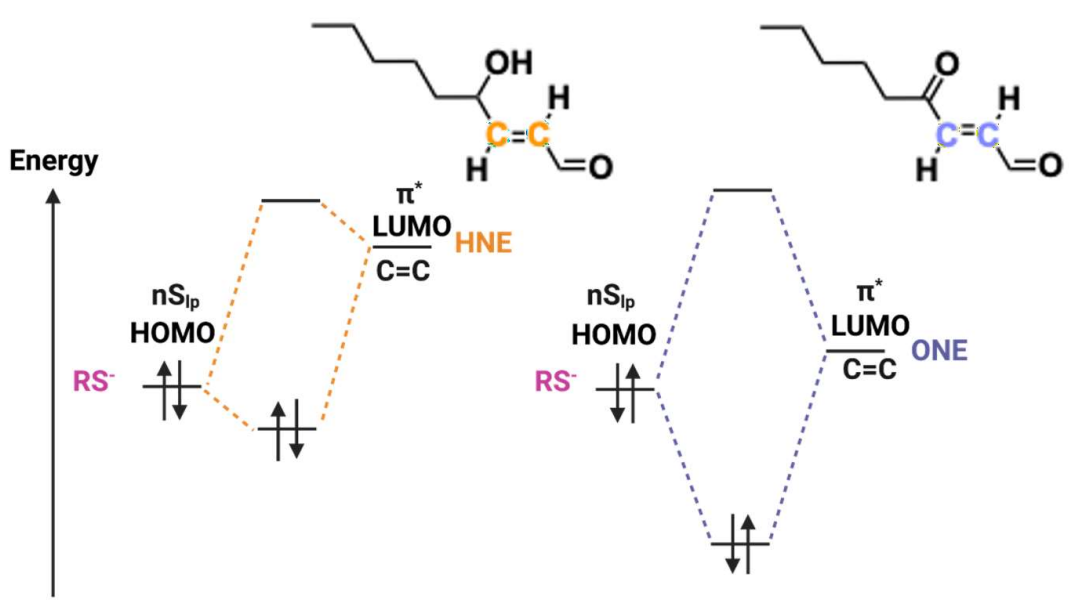

B

$\beta$-mercapto-carbony

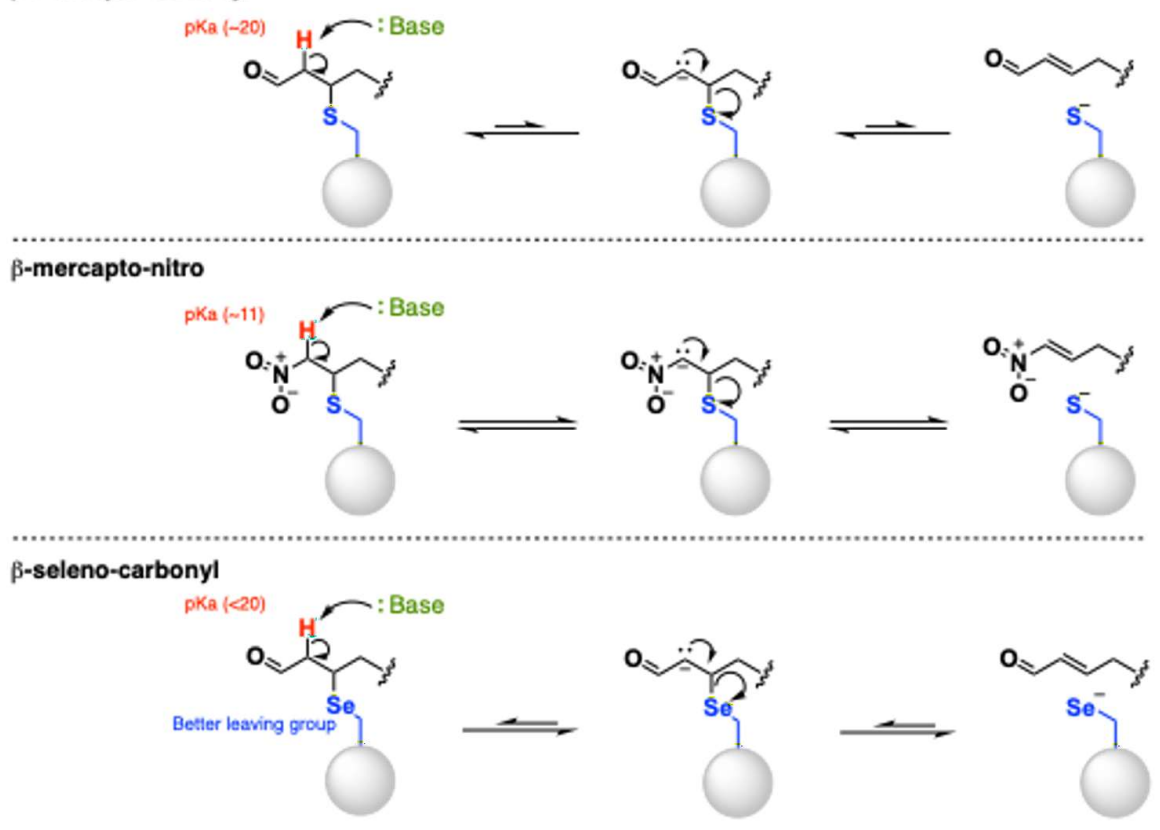

Figure 4. (A) Both 4-hydroxy-2-nonenal (HNE) and 4-oxo-2-nonenal (ONE) can react with nucleophiles, such as cysteine thiols, through 1,4-Michael addition. The LUMO of ONE is lower than the LUMO of HNE, leading to a higher reaction rate for ONE with thiolate. The above result can be ascribed to the electron-withdrawing ketone group in ONE, which creates a more positive-charge environment at the carbon $\beta$-to the aldehyde and lowers steric hinderance, making the $\beta$ carbon in ONE more prone to nucleophilic attack than that in HNE. (B) The acidic proton (in red) on the carbon $\alpha$-to the carbonyl (or nitro group in the second row) can be removed by a base, resulting in elimination of the thiol. This process depends on the $p K_{a}$ of the acidic proton. For instance, since the $\alpha$-protons of the $\beta$-mercapto-nitro species (second row) and $\beta$-seleno-aldehyde (third row) are more acidic than the protons of $\beta$-mercapto-aldehyde (first row), $\beta$-mercapto-nitro species and $\beta$-seleno-aldehyde are more prone to elimination, or retro-Michael process. Additionally, as the selenide is a better leaving group than sulfur, retroMichael process is more prone to occur in $\beta$-seleno-aldehydes than $\beta$-mercapto-aldehydes (third row). 
Fumarate, despite bearing an enedicarbonyl motif, similar to ONE and N-ethylmaleimide $\left(k_{2}\right.$ with cysteine, $\left.\sim 4000 \mathrm{M}^{-1} \mathrm{~s}^{-1}\right)^{28}$ is overall not particularly electrophilic. This is because fumarate is a dianion at neutral $\mathrm{pH}[\mathrm{pKa}(1)=3 ; \mathrm{pKa}(2)=4.5]$. These negative charges repel incumbent nucleophiles. Furthermore, forming an ionic intermediate would necessitate a formal charge of -3 to accrue on fumarate (Fig. 5A). Protonation/charge stabilization of the carboxylic acid would assuage these issues; the resonancestabilizing power of a protonated carboxylic acid is likely better than an amide ( $k_{2}$ hydroxyethyl acrylate $=20 \mathrm{M}^{-1} \mathrm{~s}^{-1}$ at neutral $\mathrm{pH}^{29}$ orders of magnitude faster than for acrylamide) (Fig. 5B). Fumarate, therefore, may display $\mathrm{pH}$-dependent electrophilic properties, being more reactive in low $\mathrm{pH}$ environments. Unfortunately, this is the environment where cysteine is most unreactive. Such concerns can be set aside in enzyme active sites, and indeed, several enzymes interact with fumarate through their active-site cysteines, such as GAPDH via Cys150¹. Other examples of this effect are HOSCN, which is a better oxidant as the $\mathrm{pH}$ is lowered from neutral to its own $\mathrm{p} K_{\mathrm{a}}(5.3)$; thus HOSCN is a particularly good oxidant of thiols that retain anionic charge at low $\mathrm{pH}$, such as enzyme active sites $(\mathrm{HOCl}$ and peroxide, $\mathrm{p} K_{\mathrm{a}} \mathrm{s}>7$, do not show this effect).

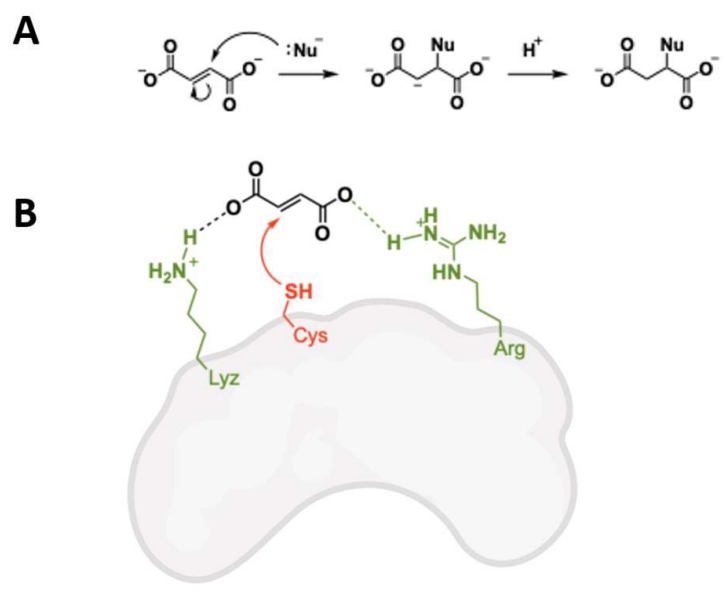

Figure 5. Fumarate as an electrophile in solution and in proteins. (A) Fumarate is a dianion at neutral $\mathrm{pH}$; nucleophilic addition to this species in solution requires formation of a trianionic intermediate, followed by protonation. Such species are likely disfavored. (B) In a protein, the one or two negative charges on fumarate may be stabilized by positive-charged amino acid side chains, e.g., lysine and arginine. The charge stabilization mitigates the negative-charge repulsion between fumarate the incumbent nucleophile and promotes electrophilicity, promoting the reaction. 
As mentioned, there is to day no enzymatic mechanism for removal of electrophilic modifications on proteins, and the vast majority of these modifications are quite stable. Thus, the main source of signal reversion is likely degradation of the modified protein. Electrophile modification of proteins has been linked to increased protein degradation, in some instances although not all ${ }^{30}$. This process is proposed to occur through unfolding. However, this destabilization effect is not well established in unperturbed cells and not known to occur on substoichiometrically electrophile-modified proteins. When destabilization effect does occur, likely it reflects hyper modification/compromise or even stimulation of cellular processes. Indeed, the rate-limiting chymotrypsin-like subunit of the proteasome (the protein complex responsible for the majority of soluble protein degradation) is positively allosterically regulated $^{31}$. Thus, unfolding of proteins, in the absence of confounding factors, would promote clearance of unstable proteins. To complicate matters more, some electrophile-modified proteins and excessive electrophile modification itself can also negatively affect proteasome activity ${ }^{32,33}$. There has been limited resolution of these quandries.

\section{Diffusion distances and clearance}

Unsurprisingly, given their diverse stabilities, these molecules in discussion display quite different diffusion distances in buffer. Overall, these are inversely proportional to their reactivity. In biological systems, the presence of detoxifying enzymes, membrane structures, and differences in $\mathrm{pH}$ can change diffusivity for most reactive species. For ${ }^{1} \mathrm{O}_{2}$, which rapidly and spontaneously relaxes to the lowest energy state of oxygen, the triplet, at a defined frequency, it is likely that diffusion distance is relatively invariable, although, diffusion distance can be prolonged in deuterated solvents. For superoxide, despite its relatively short intrinsic diffusion distance and compartmentalization, the diffusion distance is further restricted in cells by superoxide dismutase that reacts with superoxide at diffusion control. Peroxide and electrophiles, conversely, are indefinitely stable in water. However, their stabilities are significantly affected by detoxification processes. In the case of peroxide, the second order rates of clearance enzymes approach diffusion control, $k_{\text {cat }} / K_{\mathrm{m}}=10^{6}-10^{8} \mathrm{M}^{-1} \mathrm{~s}^{-1}{ }^{34}$. For electrophiles, 
detoxification enzymes tend to be less efficient than peroxide-clearance mechanisms, often having $k_{\mathrm{cat}} / K_{\mathrm{m}} \sim 10^{5} \mathrm{M}^{-1} \mathrm{~s}^{-1}$. Thus, is it often considered that electrophiles have longer diffusion distances than peroxides. Evidence exists that diffusion distance of electrophiles is larger in cells than peroxide: cells treated with external peroxide show only cytosolic increases in peroxide, whereas electrophile-treated cells show appearance of electrophile in both cytosol and nucleus ${ }^{35}$. Although, whether this is due to diffusion of modified proteins or genuine diffusion of the electrophile is unknown. Furthermore, it is unknown what the outside/inside gradient is for electrophiles, although as noted above, this is around 400-fold for peroxide and many cell types can remove of $\mathrm{H}_{2} \mathrm{O}_{2}$ effectively.

Clearance of electropiles in the cell is performed by numerous enzymes, breaking down into two camps: redox modifications and glutathione conjugation. All these activities are induced by treatment with reactive electrophiles such as $\mathrm{HNE}^{1}$, but also are lost with age, for instance, as human skin fibroblasts are passaged. This reduction in activity correlates with increased intracellular labeling of the same cells as passage number increases ${ }^{36}$. Age-dependent loss in peroxiredoxin activity has also been reported ${ }^{37}$. Thus, clearance and hence diffusion distance is likely strongly context-dependent hence it is important to use cells of low passage number, and source cell lines from reputable sources when performing experiments with reactive species.

Disulfides formed upon clearance of peroxide can also behave as an oxidant that can be used to modify specific proteins as a signaling function (Fig. 6A). Thus, processes, such as the Grotthuss mechanism, akin to what we call "changing hands" 1 , could be possible and make diffusion distances strongly context dependent. For this reason, the diffusion distance of peroxide may not represent the real diffusion distance of the information contained by peroxide. Most electrophiles are different: modifications by $\alpha, \beta$-unsaturated carbonyl species and endicarbonyls, our exemplars (Fig. S1B), cannot readily transfer between different proteins (such a transfer requires retro to reform the reactive species, or direct $\mathrm{S}_{\mathrm{N}} 2$ at a thioether, both of which are negligible) (Fig. 6B). The products of metabolism of RES (glutathione conjugates, or unsaturated acids/alcohols) are not reactive and hence do not signal covalently. 
Considering reformation of reactive species in more detail, change of hands by retro-ing of the adduct may be possible for some RES, such as unsaturated nitro-olefins (Fig. 6C), although this cannot occur through direct displacement, as it can for disulfides, and hence elaborate mechanisms would be required to shepherd the nitro-olefin to a specific protein. Thus, for proteins modified irreversibly by RES, the modified protein must behave as a signal and should carry the signal across distance if this be required. It will be interesting to observe how relevant electrophile-modified proteins can serve as signal-transfer agents and over what distances such ferrying can occur. Techniques we will discuss are poised to investigate such behaviors. Proteins are certainly less diffusive than small molecules, by perhaps 100 -fold. However, for an average protein, diffusion across a compartment of an average cell is still relatively rapid. Diffusivity across membranes, on the other hand, differs hugely for a lipid-derived electrophile and a modified protein, and thus this could well be a source of regulation. Little investigation has been done in this area. For oxidation, such mechanisms have been demonstrated ${ }^{38}$.

A

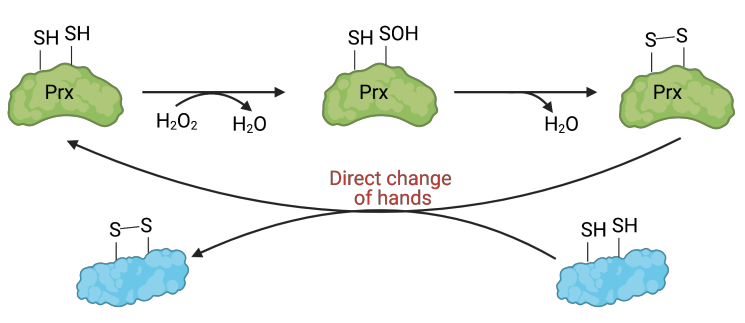

B

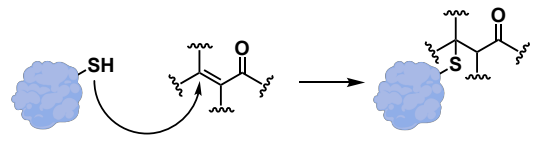

C

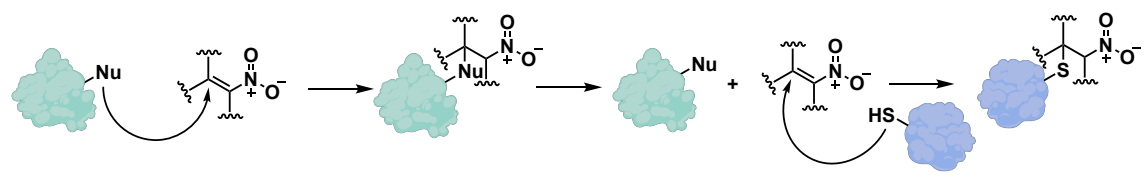

Figure 6. (A) Peroxiredoxin (Prx) can reduce the cellular peroxide, in the process forming a disulfide bond (oxidizing cysteine thiols in Prx). The oxidized form of Prx can, in turn oxidize other protein thiols. By this process, Prx can mediate signaling events. Such redox process is similar to "changing hands." Similar "changing hands" events are not immediately obvious for electrophile modifications. (B) Nitro-olefin modifications can undergo retro-Michael process, and this reforms a free electrophile, and does not readily lead to a means to selectively transfer the electrophile. (C) Modifications by $\alpha, \beta$-unsaturated carbonyl species cannot be readily undergo retro-Michael process, making 'change of hands' even less likely. 


\section{Comparisons between reaction of peroxide and electrophiles}

We have thus seen that most RES and low-reactivity ROS react sluggishly with normal thiols. Weaklyreactive molecules are in some ways ideal for behaving as chemical signals, as their rates with specific proteins, even specific residues on specific proteins, may be elevated many fold. For radicals and $\mathrm{HOCl}$, for instance, the intrinsic rate with numerous residues is already close to diffusion control, rendering significant chemical elevation in rates impossible. Such highly-reactive molecules could still use proximity effects to orchestrate protein-specific signaling, although this technique cannot readily get round inherent chemoselectivity issues, especially for molecules that react with numerous chemotypes.

From above, the reactivity of peroxide and biological electrophiles is a complex function of charge (nucleophiles are repelled by negative charges, but conjugate bases of the nucleophile are more nucleophilic), orbital interactions (lowering of the LUMO or raising of the HOMO), ability to form a stabilized intermediate during adduction (enolate stabilization), and (de)solvation. We can compare nucleophilic attack on peroxide and unsaturated carbonyl bearing species and consider transition-state requirements to examine if one transition state be sufficiently similar to the other promoting similarities in modification efficiencies. As a nucleophile starts to attack the $\sigma^{*}$ of the peroxide, or the $\pi^{*}$ of the unsaturated carbonyl, significantly-different transition states arise (Fig. 7). For peroxides, there is a traditional $S_{N} 2$ transition state, proceeding through a trivalent oxygen atom, the nucleofuge being hydroxide, or water, diametrically opposed to the incumbent nucleophile, on the apical portions of the trigonal bipyramid (Fig. 7A). Conversely, attack of the enone occurs through a tetrahedral transition state, through attack at the Bürgi-Dunitz angle on an s-cis or s-trans conjugated system. Electrons move into what is essentially a planar allylic anion. The highest coefficient of charge localizes to the oxygen atom, but this is several atoms remote from the site of nucleophilic attack. There is partial negative charge build up on the $\alpha$-carbon atom, although the carbonyl carbon atom exists on a node (Fig. 7B). In both instances, protonation, and stabilization of the intermediate is required to promote rates. However, aside from the geometrical differences, in case of attack at peroxide and the electrophile, 
this must occur at drastically-different positions. Furthermore, the peroxide transition state is scissile, whereas the addition to electrophiles does not change the connectivity of the carbon skeleton. Even among $\mathrm{S}_{\mathrm{N}} 2$ transition states, these can vary widely. For instance, many reagents used to identify generally reactive cysteines use $\alpha$-chloro carbonyl reagents as surrogates. Nucleophilic addition to such reagents likely occurs through a transition state that involves interaction with the $\pi^{*}$ orbital, which in the case of ketones can lead to an alkoxide product that undergoes a 1,2 shift to give the product; such a trajectory does not ideally mimic addition to peroxide either, as there is negative charge build up at two positions. Thus, it is critical not to assume that a reactive thiol is indiscriminate; the more selective a thiol be, likely the more reactive with its chosen substrates it may be ${ }^{1,39}$.

\section{A}

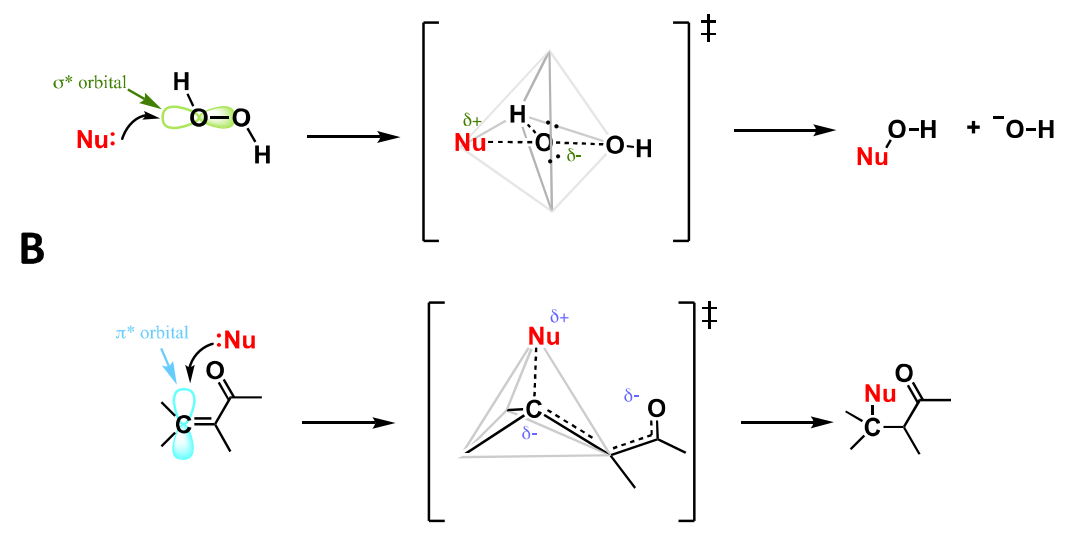

Figure 7. Differences in transition states for peroxide and enones. (A) For addition to peroxide, the nucleophile attacks the $\sigma^{*}$ orbital of the peroxide $\mathrm{O}-\mathrm{O}$ bond, proceeding through an $\mathrm{S}_{\mathrm{N}} 2$-type substitution. A trigonalbipyramid transition state forms in the reaction, as shown. (B) The nucleophile attacks the $\pi^{*}$ of the unsaturated carbonyl, at the Burgi-Dunnitz angle. A tetrahedral transition state forms, in which the $\beta$ carbon to the carbonyl is in the center. The partial negative charge on central carbon delocalizes to the oxygen of the carbonyl group, which stabilizes the transition state.

\section{Experiments that seek to compare cellular or whole-organism properties of RES and/or ROS}

It is often instructive to compare labeling, phenotypes, and toxicity profiles of electrophiles and oxidants, such as those described above, in cells. Although this can lead to useful insights in terms of gross toxicity, etc., we stress that the extreme differences in chemical makeups of these molecules 
renders such comparisons, e.g., at the proteomic level, of hugely limited insight. This is because localization/permeation in the cell, and hence reactivity therein may be intrinsically biased. When comparing all but the most general biological activities, it is therefore important to have a keen understanding of the chemical attributes of the molecules being compared. For example, dimethyl fumarate (DMF) is an approved drug, whose structure is based on the natural metabolite fumarate. DMF is uncharged (hence likely permeates cells, and indeed tissues post oral ingestion, differently to fumarate). DMF is also significantly more electrophilic than fumarate. Monomethyl fumarate (MMF), an approved multiple sclerosis drug, that is 30 -fold less reactive with glutathione than $\mathrm{DMF}^{28}$, and a metabolic product of DMF, is intermediate between fumarate and DMF in reactivity and charge, and once again likely has particular permeation and distribution properties. However, comparisons between these molecules by external bolus dosing to cells, continue to be reported with few or no considerations for key divergences in permeation, stability, and the like. Of course, once in the cell, DMF may well rapidly hydrolyze to MMF, or this could occur post adduction of DMF to proteins, to give apparent MMF products; indeed, primary amide hydrolysis in active sites has been reported ${ }^{28}$. Thus, the route taken for "MMF" to arise in the cell/on a protein due to DMF treatment, is not the same as the route taken for externally bolus-dosed MMF. Considerations for regioselectivity of addition and careful in vitro experimentation could be hugely beneficial for the field; for instance, the concept that hydrolysis of an ester can impact the electrophilicity of $\alpha, \beta$-unsaturated species has been applied to produce 'kinetically selective covalent inhibitors' ${ }^{\prime 0}$.

\section{Detection}

Our abilities to detect RES and ROS differ significantly. Although a generalization, the detection of peroxide has benefited from the increased understanding/exploitation of protein-based sensors that function "chemoselectively". In addition, the reversibility of peroxide-mediated oxidations overall renders ROS more suited to make measurements at equilibrium. Equilibrium measurements in turn give the ability to measure changes in real time, obviously limited by the kinetics of oxidation and re- 
reduction and diffusivity of the specific small-molecule-based detection agent. For protein-based peroxide sensors, $k_{2}$ oxidation is rapid $\left(\sim 5 \times 10^{5} \mathrm{M}^{-1} \mathrm{~s}^{-1}\right)^{41}$, relative to most redox-controlled biological processes. Many peroxide measurements systems also function ratiometrically, allowing absolute readouts to be achieved. Overall, the peroxide concentration in cells is likely in the mid-to-low nanomolar range ${ }^{1}$; in blood plasma concentration is around $1-5 \mu \mathrm{M}^{42}$. This ratio resembles the approximate outside/inside gradient for extracellularly dosed peroxide (400-fold). Genetically-encoded sensors can also be expressed in specific cellular locales easily, so we have gained insight into subcellular localization of peroxide ${ }^{41}$. Nevertheless, given uncertainties in peroxide diffusion distances, it is unclear what resolution is needed to really understand subcellular peroxide so there is work left to do in this area. Some protein-based sensors of ROS other than peroxide are known, but these are less well developed. Unsurprisingly, few of these non-peroxide sensors are entirely chemoselective ${ }^{1}$. Small molecule-based sensors of ROS have also been reported, including boronates for peroxide, hypochlorite sensors, and spin traps for radicals ${ }^{1}$. Ratiometric versions are known, but these are most commonly turn-on and chemoselectivity is rarely ideal. Nevertheless, it should be noted that ROS are typically not present as single entities, so claims of a specific ROS behaving as a specific signal need to be met with caution. All sensors, including protein-based sensors, may be influenced by changes in unrelated variables, such as $\mathrm{pH}$. Many of these Achilles heels are now known, and hence careful research can very much aid planning. Robust practices will try to account for unforeseen issues. Thus, all data derived from probe-based measurements, require careful interpretation and should deploy numerous chemically-divergent probes to build up a consistent picture.

RES concentrations in cells are either measured directly by HPCL or via ELISA of lysates ${ }^{43}$ or assessed using irreversible chemical probes, often analyzed post cell lysis, rendering measurements timedependent, likely relatively slow, and prone to variation. Clearly these methods can also give little information about sub-cellular locale, particularly as most RES can diffuse through membranes during lysis/organelle extraction. There is no commonly-used measurement that can report on changes in endogenous RES in real time. Few, if any, RES measurements are ratiometric, rendering concentrations 
obtained hard to standardize. Thus, we are overall quite clueless as to the local cellular concentrations of RES, whereas we have some reasonable idea of local peroxide concentrations. It is practically easier to measure concentrations of RES in blood serum. Quoted values range around $0.1-10 \mu \mathrm{M}^{1}$, which is disappointingly varied. However, the measured serum concentrations of, e.g., HNE, is impacted by not only by method of detection, but also by biological context e.g., lifestyle, health and age. Thus, it is unclear how much the disparity between quoted values reflects actual biological diversity as well as methodological differences. Given that HNE is freely diffusible across membranes, and ignoring cellular detoxification strategies, the values of HNE in blood set an expected range for overall cellular HNE concentrations. Consistent with this thought, global cellular values of 0.1-10 $\mu \mathrm{M}$ are often quoted for $H N E^{1}$.

\section{Exchange detection methods}

Innovative detection methods are arising regularly. One recently reported method takes advantage of the ability of reactive species to interconvert ${ }^{44}$, specifically by converting peroxide to an ephemeral electrophile species that can label nearby proteins irreversibly. In this sort of assay, arguably the equivalent of a traditional coupled assay, the rate of interconversion of the measured species with the probe should be rapid and chemoselective, and the product of the conversion should have a half-life many folds shorter than the molecule intended to be measured. Thus, low concentrations of analyte can be measured rapidly, and accurate locale-specific measurements can be achieved. Exchange approaches are compatible with mass spectrometry, as well as fixed-cell imaging (i.e., detection of endogenous proteins proximal to the stress through immunofluorescence). In an approach recently reported, peroxide was used to activate a latent electrophile appended to a dye (Fig. 8). The electrophile thus formed was proposed to label proteins in proximity to the peroxide. The chemistry proceeds via oxidation of a pinacol borane, via formation of the "ate"-species and an aryl shift, similar to turn-on fluorescence boronate probes ${ }^{1}$. The resulting phenoxide can then eliminate a pendant fluoride to form an o-quinone methide, which is trapped out by thiols. Critically, the overall 
transformation is to convert information in the form of peroxide, a transient signaling molecule, which can be hard to measure, especially in tissue slices, to an electrophile-tagged dye, which can indelibly modify proteins. Using this strategy, evidence was provided for an increase in peroxide in activated macrophages.

This approach provides a level of plasticity unavailable to real-time sensor methods. It will likely be useful, particularly for measuring low peroxide concentrations, or where very precise measurements of peroxide localization, are needed. However, it is worthwhile noting that the derived electrophiles are almost certainly chemoselective, i.e., that they will favor reactivity with specific proteins, and may have divergent stabilities across different environments. How this affects resolution, protein specificity, and signal is complex and needs care. Furthermore, this molecule showed off-target detection of isolobal reagents, such as pernitrate, although hypochlorite was reported to not be detected. It further remains how modification of proteins by the liberated electrophile may impact signaling. Other drawbacks were a pH dependence of labeling, and a relatively large diffusion distance of the probe, although the latter is difficult to calculate in vivo and could well be significantly less than what was reported $^{44}$. Further synthetic development of probes, with reduced diffusion distance, broader reactivity, etc., based on this initial design could also bring improvements.

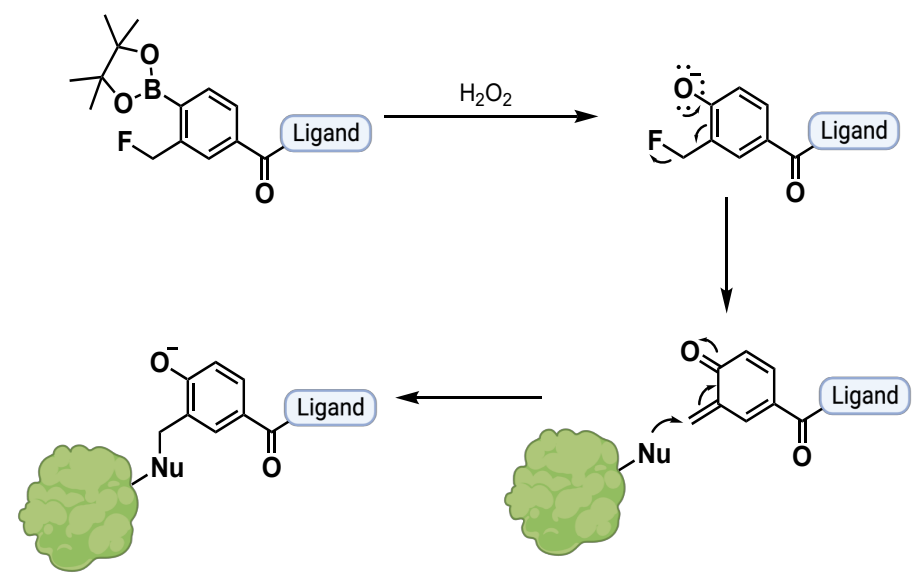

Figure 8. A probe that converts peroxide to an electrophile that labels proximal proteins to give high locale specificity and sensitivity. 
Detecting ROS- and RES-dependent post-translational modifications (PTMs)

Given that reactive species principally manifest their influence through reaction with specific proteins, significant effort has been expended identifying specific modified residues, on specific proteins. Indeed, as location and context are hugely important for ROS, and the sensors themselves are not ideal, one could readily argue that quantifying PTMs is a more relevant proxy for how a specific stress upregulation affects cellular behavior. Such an argument relies on the fact that sensing of the modification can occur under physiologically-relevant conditions, and that physiologically-relevant level of PTMs can be detected by the technique in question. Indeed, a large number of "chemoselective" detection methods now exist. In fact, the majority of the methods do not directly read out the PTM, but convert the PTM to a "specific" chemical entity whose loss or gain is assayed. Such methods come with a side helping of caveats (e.g., chemoselectivity? what conditions are they functional? How do off-target effects of probes intersect with conditions?) that are often neglected or intrinsically non-addressable.

\section{Antibodies}

Antibodies have been used to detect specific PTMs directly by western blotting, or in combination with mass spectrometry (MS). These can be used to investigate modifications of a specific protein or at a proteome level. As with all antibodies, there are issues with non-specific effects and lack of proteome coverage; many antibodies also require oxidizing environments and have relatively slow-binding kinetics. Traditional antibody-based validation techniques are also not ideal to parse how selective redox-sensitive antibodies are.

\section{Mass spectrometry (MS)}

In general terms, MS is the only diagnostic means to determine if a precise PTM has occurred on a specific residue in an otherwise unaffected system. This method has the ability to define which specific residue is modified and the precise chemical makeup of the product formed. However, MS has numerous limitations that render it far from apposite for understanding biological relevance. The 
method is lengthy: numerous processing steps need to be conducted in lysed/partially-purified samples, during which time oxidation, retro-modification, or conversion of the modified protein/peptides could occur. Innovative strategies have been deployed to try to account for such behavior on specific proteins, such as doping lysates with exogenous $\mathrm{N}^{15}$-labeled recombinant proteins, made in $E$ coli. However, recombinant and native proteins are not always the same in terms of modifications, tags, and cleavages/splicing. How such differences affect oxidation is rarely known ${ }^{45}$. Alternatively, saturation with heavy oxidants post lysis (e.g., $\mathrm{O}^{18}$-peroxide) has also been used, although this treatment clearly also is not ideal and could compromise sample integrity ${ }^{46}$. However, the biggest issue with native MS is that the signal observed for different forms of the same peptide, e.g., in the oxidized or reduced forms, is severely affected by the stability, volatility and ionization capacity of each form (these are often erroneously or negligently assumed to be "equal"). Necessarily, therefore, detection of modified forms of a peptide is dependent on compatibility of each form of that peptide with the MS method used; thus this method is prone to false negatives. A related but more fundamental point is that to understand biological relevance, ligand/PTM occupancy should be determined. But due to variation in ionization, volatility, stability, and pleiotropism of residues modified by reactive molecules, direct MS measurements cannot give quantitative insights, rendering PTM detection by MS unable to inform on the extent to which a biologically relevant amount of a particular species is present. Coupling MS with 2-dimensional gel electrophoresis, for some oxidative PTMs at least, may allow some improvement, but 2D-gels are non-quantitative. SILAC or TMT methods are also potentially useful, but as one oxidized residue can give spawn manifold variants of oxidized/electrophile-modified states, such approaches do not really inform on product distribution either ${ }^{47}$.

To assign relevance of an PTM by a reactive molecule, careful investigations require independently supporting the detection of a modified residue with mutagenesis studies. Yet, as the number of different potential variants of PTMs derived from a specific RES/ROS on a protein is legion, simply determining that a loss of a residue proposed to be a sensor of a specific RES/ROS suppresses a phenotype is also uninstructive: even if the mutation impacts the response to an oxidative-stress 
stimulus, the nature of the chemotype triggering the cellular response, and also the chemicallymodified proteoform so produced in cells remain unproven. Concerns are even more elevated if the protein of interest be a non-essential gene, where the mutant is not assayed for activity, or not shown to complement a specific phenotype due to loss of function of the allele in question. Furthermore, most redox-linked PTMs do not have direct mimetics that can be created through mutagenesis, like the use of aspartate to mimic phosphorylations for instance. Sulfenic acid may be mimicked through mutation of cysteine to serine, but this change is far from ideal, for instance. Redox signaling further may in some cases have high residue redundancy, which may complicate analysis, as it has been shown to in the case of ubiquitination-dependent signaling ${ }^{48}$.

\section{Molecular probes}

Given the above issues, measurements diagnostic for a specific chemotype that provide a quantitative analysis of labeling, are crucial. As oftentimes these methods can be deployed directly in cells, or immediately post lysis, issues of labeling during processing are assuaged relative to direct MS methods. Molecular probes have been developed for a fleet of PTMs by reactive molecules. We will discuss some of the more recent ones, and for others refer readers to a previous review ${ }^{1}$. Before we discuss these methods, it is worth bearing in mind that the issues with indirect methods to measure a modification are different from direct detection methods, but are also significant. The first issue is that the modification is not directly assessed. Typically, loss of a signal due to the free cysteine, or increase in a chemically-modified cysteine, is detected. Thus, data are prone to mis/over-interpretation. It is important to understand how the probe was validated and in what systems have rigorous validation data been provided. Contrary hypotheses should also be researched. Oftentimes, assumptions of probes behaving "selectively" are based on extrapolation from simplified/less-relevant systems, despite there being manifold possible reaction pathways for even ostensibly simple reagents ${ }^{49}$. The second issue is coverage. There are approximately 200,000 unique protein-cysteines in humans ${ }^{23}$. Molecular 
probes often pick up only a fraction of those present, clearly hampering understanding of reactivity and the importance of the PTMs identified (if a molecule be shown to affect 3 cysteines out of 10,000 detected by a specific method, extrapolation implies that the molecule should label 60 total cysteines). It is thus crucial that complementary probe-based methods be developed, and fair consideration be given to methods that ostensibly measure "the same" PTM. Application of/validation by numerous approaches in tandem, including multiple probes where applicable, likely gives the most robust conclusion. Indeed, in addition to the chemical probes, mutated proteins can also be used to identify modified proteins as we will see below.

\section{Dimedone}

Dimedone is for better and worse the prototypical molecular probe (Fig. 9A). This reagent, which was reported to react with sulfenic acids around 50 years ago ${ }^{50}$, has transcended chemical biology and has entered the biological canon. Indeed, the number of publications using this reagent elevates each year. The currently-accepted consensus is that the dimedone anion can displace $\mathrm{S}-\mathrm{OH}$ forming an irreversible carbon-sulfur bond. It is unlikely that dimedone reacts with sulfinic or sulfonic acids. Thus, ignoring other oxidized thiol species, sulfenic acids formed in biological systems can be chemoselectively tagged for downstream processing. Dimedone is a relatively strong organic acid $\left(p K_{a} \sim 5\right)$, by virtue of it possessing a methylene function straddling two ketone groups. Thus, unlike many carbon-based nucleophiles, the dimedone anion, which is the active form, can be deployed in mild aqueous conditions. Perhaps unsurprisingly, given the stability of the anion, this reagent has a relatively sluggish reaction rate with sulfenic acids $\left(\sim 5 \mathrm{M}^{-1} \mathrm{~s}^{-1}\right)$. 100-fold improvements to this rate have been achieved, by destabilizing the enolate, promoting nucleophilic addition (raising the HOMO). However, it should be stressed that this is on a model substrate and rates of reaction between dimedone and different protein sulfenic acids can vary by at least $\sim 1000$ fold ${ }^{51}$, and fold improvements for one substrate do not necessarily correspond to improvements for all substrates. 
One of the most important aspects of dimedone is that it can be functionalized with a specific chemical handle, for instance a dye, or an azide, while retaining its reactivity and selectivity (at least in model substrates). Thus, sulfenic acids can be directly converted to a fluorescent, or bioorthogonallyfunctionalizable group. This allows, e.g., visualization, affinity detection. For instance, 1-(pent-4-yn-1yl)-1H-benzo[c][1,2]thiazin-4(3H)-one 2,2-dioxide (Fig. 9B), a probe that reacts with sulfenic acids 200 times faster than dimedone, was used to profile Arabidopsis proteins oxidized in response to $100 \mu \mathrm{M}$ bolus peroxide treatment. This study identified 1194 proteins previously not known to be susceptible to oxidative stress. Intriguingly although 1-(pent-4-yn-1-yl)-1H-benzo[c][1,2]thiazin-4(3H)-one 2,2dioxide is significantly more reactive than traditional dimedone reagents, and overall covered more cysteines, the traditional dimedone reagent, combined with a genetic sensor of sulfenic acid, YAP, were still able to identify 212 proteins not covered by 1-(pent-4-yn-1-yl)-1H-benzo[c][1,2]thiazin-4(3H)-one 2,2-dioxide ${ }^{52}$. 1-(Pent-4-yn-1-yl)-1H-benzo[c][1,2]thiazin-4(3H)-one 2,2-dioxide was also used to implicate CD36 signaling in promoting oxidation of Src kinases. This oxidation was proposed to stimulate this family of kinases ${ }^{53}$. In another recent paper, the circadian pacemaker protein, CLOCK, was proposed to be regulated by formation of sulfenic acid in part based on a proteomics experiment that leveraged a dimedone-based probe. Based on these results, activity of CLOCK was linked to C195-specific oxidation that was proposed to occur through the function of $p 66^{\text {shc }}$.

A

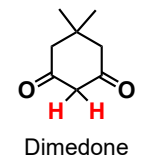

B

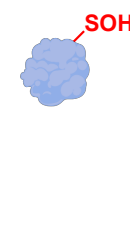

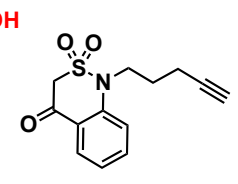

BTD
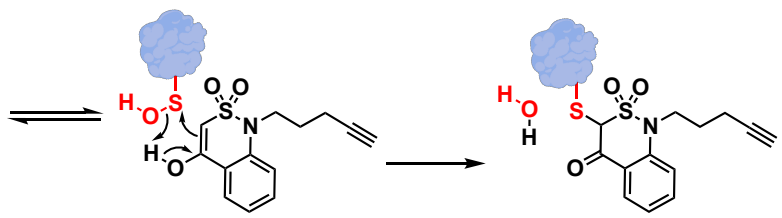

Figure 9. (A) Structure of dimedone. Due to the two electron-withdrawing carbonyl groups, the red-colored protons are acidic. (B) 1-(pent-4-yn-1-yl)-1H-benzo[c][1,2]thiazin-4(3H)-one 2,2-dioxide (BTD) probe. The enolform BTD can covalently modify proteins containing sulfenic acid groups. The orthogonal group on the BTD, in this case an alkyne, can be utilized for subsequent proteomics analysis or other applications. 
The dimedone chemoselectivity problem

It should be noted that the chemistry of sulfur is complex, and very much divergent from elements in Period 1 and 2. Sulfenic acids can unsurprisingly occur in multiple forms in biology, including as hypervalent species, which may react unexpectedly with dimedone. On the other hand, dimedone can label both selenocysteine selenenic acids and selinic acids ${ }^{54}$, although these modifications are unstable to direct displacement intramolecularly, and the latter also to Pummerer type reactions in model substrates. This labeling extent is therefore likely minor compared to the sulfenyl proteome, assuming model substrates be representative of such adducts. However, there are also growing reports that dimedone can label other oxidized forms of cysteine, principally sulfenylamides (Fig. S1D), that give identical products to those formed if a sulfenic acid were the starting point. Reaction with carbonyls, and other oxidized carbon species, are not inconceivable, even if model systems show such rates to be slow, and these were not detected in MS analysis ${ }^{55}$, of albeit a limited number of thiols, after 15 min treatment with $8 \mathrm{mM} \mathrm{DTT}$ at $75{ }^{\circ} \mathrm{C}$. Taken together, detecting dimedone labeling cannot be seen as proof that a sulfenic acid be formed. Therefore, dimedone's real utility is in assigning changes to specific residues, and biochemistry and other more traditional methods are needed to assign the specific modification in question. Indeed, this leaves dimedone as a useful and applicable reagent anyway.

Persulfidation detected by dimedone switch technology

Protein persulfides are proposed to be generated through nucleophilic displacement of sulfenic acids by $\mathrm{H} 2 \mathrm{~S}$. The reasons for this process, are coming into light due to work from several laboratories. However, this is a thorny problem. Disulfides in water are expected to have intrinsically have high nucleophilicities relative to thiols. This is based on analogy to peroxide and model persulfides that are more nucleophilic than water or the corresponding thiols respectively. However, the situation is complex. In the gas phase, peroxide is less nucleophilic than water, as should occur because the oxygen anion in peroxide is significantly less electron-rich than that in hydroxide; it is indeed the desolvation of the peroxide anion in water relative to the hydroxide anion that is the principal source of nucleophilicity 
of peroxide. Dominance of solvent effects are common in real chemical systems, and have been proposed to explain why o,o-ditertbutyl phenoxides are significantly more basic than the corresponding phenols, although these differences wane as the basicity of the phenoxide anion decreases ${ }^{56}$, presumably because the charge density is less oxygen localized. Thus, it is unclear what the properties of a persulfide relative to a thiol may be in a protein active site, or near the surface of a hydrophobic pocket and how predictive different model systems would be. Clearly, this is likely to be context dependent. Furthermore, in many protein active sites, thiol nucleophiles are deprotonated, and hence the enhanced $p K_{a}$ of the disulfide relative to the thiol is also likely to be less relevant. Clearly this argument does not discount the utility of persulfidation, nor that it exists in cells, but it means that context could be particularly important for protein persulfidation and nuances of chemistry must be appreciated if we are going to understand what this modification does.

As with many problems in the field of redox biology, deriving such understanding starts with defining proteins endogenously susceptible to persulfide formation and understanding how persulfide forms in cells. Recently, a means to detect protein persulfides was proposed. This method used 7nitrobenzofurazan to label a host of nucleophilic residues, including thiols, sulfenic acids and persulfides. It was then proposed that dimedone treatment was only able to displace the persulfide adduct by reference to model systems. The dimedone-labeled cysteines could then be detected by fluorophore/biotin click. One attractive aspect of this strategy was that the global proteome was labeled with nitrobenzofurazan, meaning that protein load could be detected by examining fluorescence due to nitrobenzofurazan. Although this nitrobenzofurazan-derived signal was not always consistent. Nevertheless, dependence of the signal ascribed to persulfide formation was shown on cystathionine lyase, a protein believed to be responsible for $\mathrm{H} 2 \mathrm{~S}$ formation.

\section{Detection of RES modifications on proteins}

In principle, detecting electrophilic modifications should be "easier" than detecting oxidations. However, there are many similar electrophiles formed in biological systems. In many instances, the 
adducting molecules may differ through a single methylene homologation, or a pendant hydroxy group. Such species are hard to distinguish using antibodies or chemical derivatization. Perhaps worse, many electrophiles are bi-functional: 4-hydroxynonenal can undergo attack at the terminal carbon of the enone, or direct addition to the aldehyde. Post addition to the enone, the remaining aldehyde can form an aldimine post adduction of cysteine via the $\alpha, \beta$-unsaturated olefin, or potentially react with dimedone: conversely, formation of an unsaturated imine changes the electronic properties of the electrophile and could change reactivity. More importantly, with $y$-hydroxylated electrophiles, the products of thiol Michael adduction can in turn form cyclic hemiacetals, dehydrate, and even aromatize post adduction. All these products have different structures that may not be identified by an "HNE" antibody, and they also have different masses and reactivities. To make matters worse, redox chemistry of pendant aldehydes of proteins is also often reported and this process is catalyzed for enzyme adducts $^{57}$. For these reasons, antibody detection of electrophile modifications, particularly lipid-derived electrophiles, is now avoided.

\section{Mass spectrometry}

This method is also considered to be the "gold standard" for detecting modification of a specific residue, despite suffering from most of the abovementioned issues. The protean nature of electrophiles further erodes MS's quantitative accuracy and enhances MS's proclivity towards false negatives ${ }^{58}$.

\section{Activity-based profiling methods}

Activity-based protein profiling methods have been used to detect modification of specific cysteines by alkylating agents. In its original guise, iso-TOP-ABPP functioned like an enzyme activity assay in that it measured loss of labeling by a probe as a proxy for how much labeling by a specific reagent occurred (Fig. 10). Such methodologies work well in vitro, and when using specific substrates with diagnostic outputs of product in cell lysates. However, as iso-TOP-ABPP does not have these virtues, its reliability is relatively low, compared to enzyme-activity-based ABPP probes. Furthermore, although improvements to this method have been made, the coverage of iso-TOP-ABPP is low $(<10 \%$ 
cysteome $)^{59}$. Newer methods that are variants on this theme have arisen in the literature. For instance, a method to detect protein modification by lipid-derived electrophiles via aldimine derivatization of the aldehyde residual post conjugate addition to lipid derived electrophiles (such as HNE) has been released $^{60}$ (Fig. 11). This method directly detects modified proteins, which is an important step. However, it should be stressed that protein carbonyls may be reduced/oxidized post conjugation (leading to false negatives) and, as we saw above, protein carbonyls can be made by a host of factors not related to modification by reaction with electrophiles. Furthermore, when used to detect sensors in lysates, where proteomes are first treated with an electrophile then the alkynyl-substituted amine, or even to detect endogenous HNE in cells, it is possible that the reactive species be the HNE iminium ion and not HNE itself. 
A

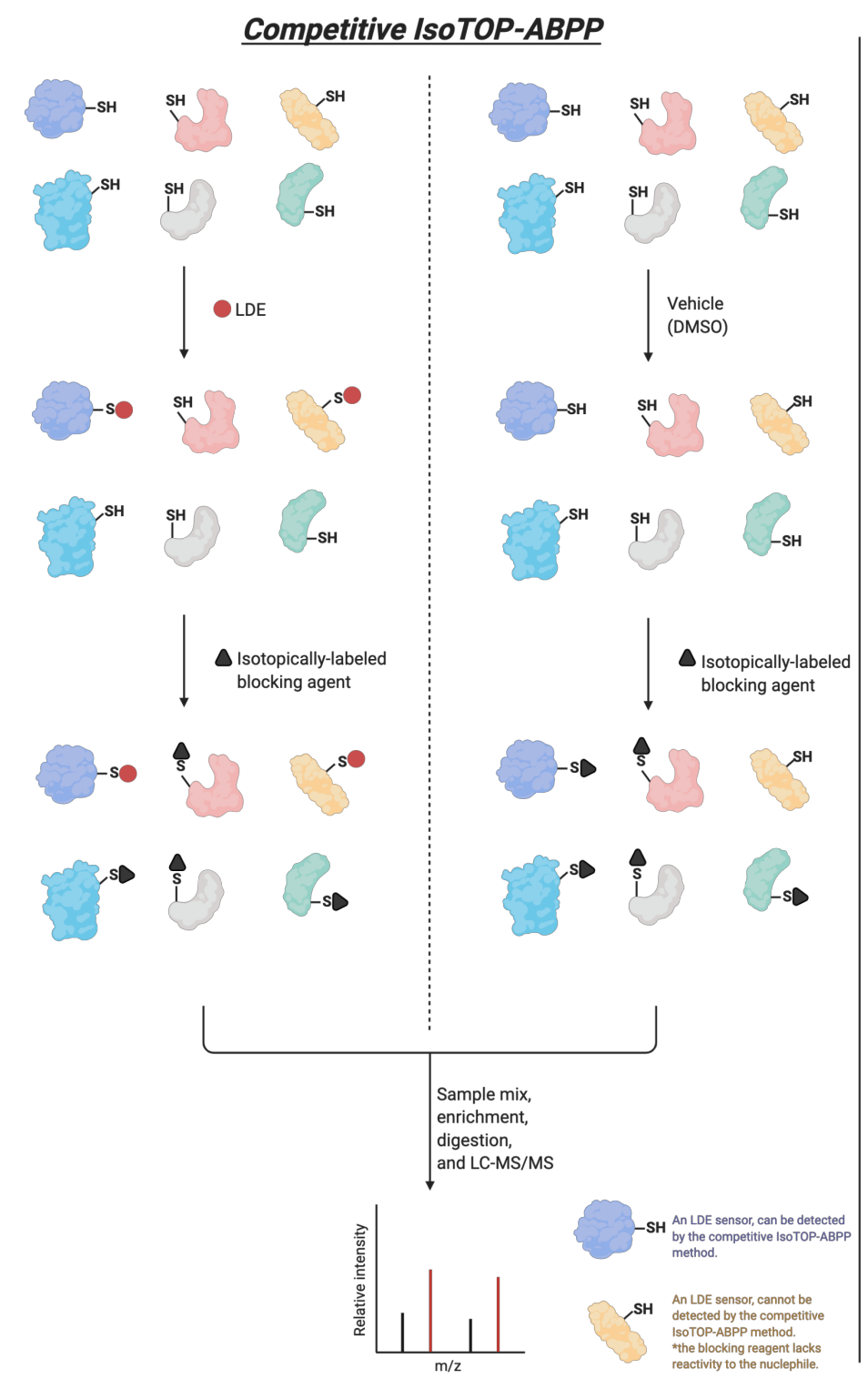

B

\section{Aniline-derived Probe}
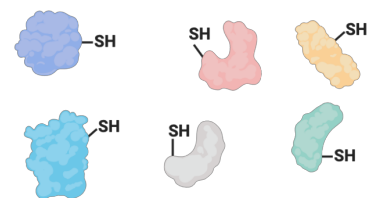

$\downarrow$ OLE
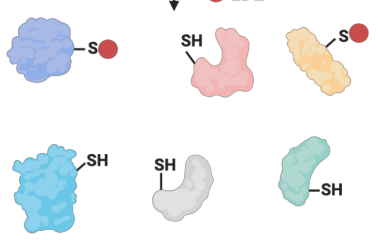
acetylene (APA)
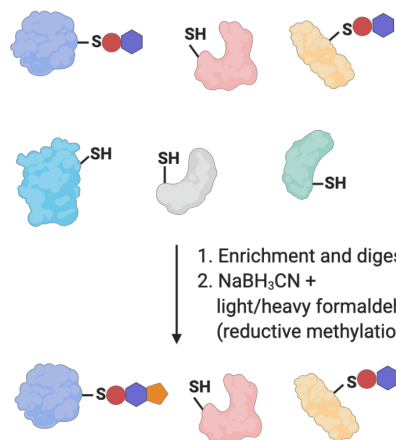

1. Enrichment and digestion 2. $\mathrm{NaBH}_{3} \mathrm{CN}+$ light/heavy formaldehyde (reductive methylation)

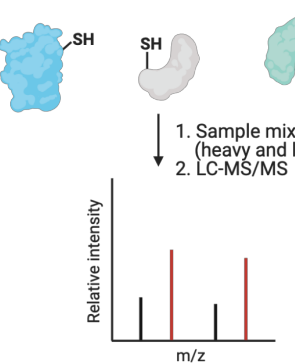

Figure 10. (A) Competitive IsoTOP-ABPP. Experiment groups are treated with an LDE of interest (red circle), while the control group is treated with vehicle, e.g., DMSO. To both groups, an isotopically-labeled blocking agent (dark triangle) is subsequently added to globally label remaining nucleophiles. Samples from both groups are mixed (equally), enriched, digested, and analyzed by liquid chromatography-tandem mass spectrometry (LC-MS/MS). From the signal loss of isotopically-labeled protein, the LDE sensitivity of proteins can be quantitatively ranked. Several reports have shown that depending on the nature of the blocking agent (e.g., iodoacetamide vs. Nethylmaleimide), different hits are scored. This is because different blocking agents label different sets of proteins, and proteins that cannot be labeled by the blocking agent in question, will not be scored as hits even if they can be labeled by protein. This scenario is illustrated for the target protein in orange. In addition, blocking agent may not necessarily bind to the same residue/site as where LDE binds, leading to additional potential false negatives. (B) Aniline-derived probe. Biological samples are treated with LDE of interest, followed by the treatment of aminophenyl acetylene (APA) probe. Subsequently, the samples are enriched and digested before the isotopic labeling through reductive methylation (see also Fig. 11). Samples with different isotopic labels are mixed, and subjected to LC-MS/MS. 


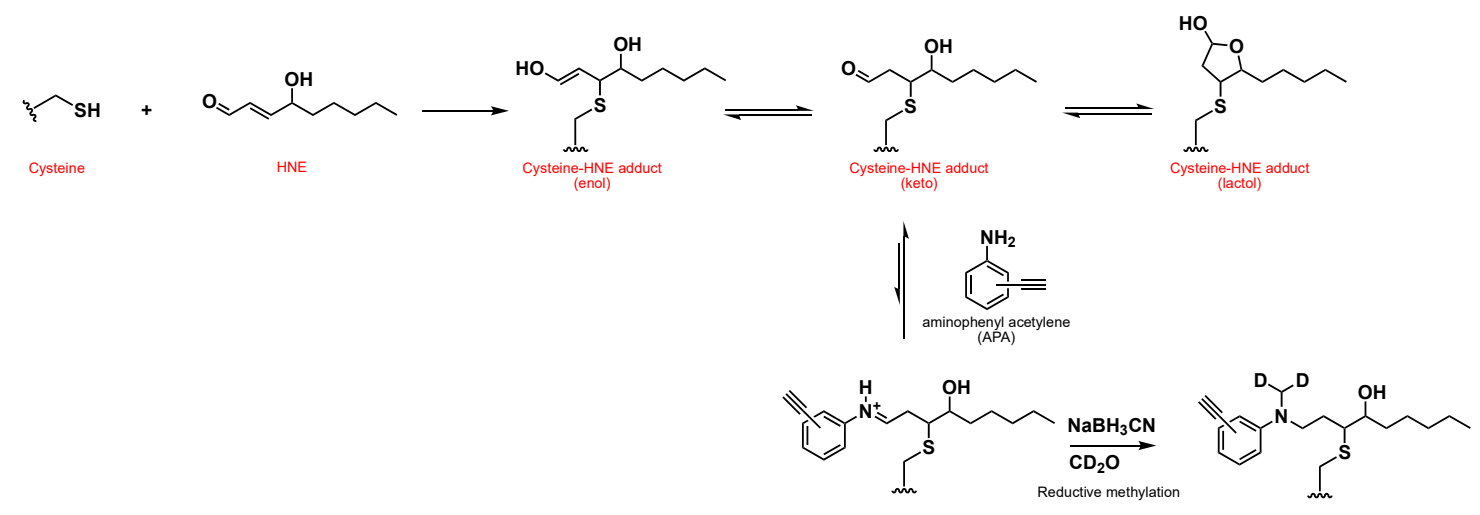

Figure 11. Cysteine residue can react with HNE to form the cysteine-HNE adduct, which can tautomerize between enol and keto. The aldehyde-form of the adduct can also be in equilibrium with lactol (formed via intramolecular cyclization between the aldehyde and the alcohol). Aminophenyl acetylene (APA) probe (see also Fig. 10B) can form an imine with aldehyde-form of the cysteine-HNE adduct, which can be reduced by sodium cyanoborohydride and form covalent linkage with isotopically-labeled formaldehyde (known as reductive methylation labeling). The APA probe can also be applied to other LDEs with $\alpha, \beta$-unsaturated carbonyl group.

\section{Profiling through chemical modifications}

One chemical aspect that separates electrophiles from oxidants is that post adduction of an electrophile to a protein, often a hydrophobic tail from the electrophile is introduced. Thus, when a protein is modified by an electrophile, there is often some functionalizable chemical space added to the protein. This has been taken advantage of in both sensible and occasionally less-sensible ways. For lipid-derived electrophiles, e.g., HNE, the simplest structural modification that can occur is conversion of a terminal ethyl group on the electrophile to an alkyne. This very minor modification, which has not yet ever been shown to change the selectivity of a lipid-derived electrophile, allows biorthogonal modification. Of course, it must be ceded that conversion to the alkyne changes the properties of the electrophile, and that change, albeit small, could affect biological function considerably. Nevertheless, for HNE, the corresponding alkyne version is often used. Several authors have tried to perform experiments using fluorophore or otherwise tagged HNE and other lipid-derived electrophiles. This approach is best avoided. On the other hand, our other representative electrophile, fumarate, is less amenable to alkyne functionalization. One could consider converting one ester to the corresponding alkynyl amide, although we know that changes the reactivity of the system and the charge somewhat. The drug 
Tecfidera, dimethylfumarate, may be more amenable to this modification. The electrophilicity of an ester and an amide are, admittedly, not equivalent but they are close; sterically a methyl group and a propargyl group are also similar, especially as both exist in the $s$-cis conformation. ABPP has been used to investigate dimethyl fumarate targets, with mixed success. Of course, the great benefit of ABPP experiments is that the electrophile can be deployed without any modification. Although this comes with the price of not knowing the nature of the modification.

To deal with numerous of the issues above, we proposed a new method that we dubbed G-REX - global reactive electrophiles and oxidants (Fig. 12A and C). This method harnesses alkyne functionalization of an electrophile, aiding direct detection of a specific modified protein. However, it also uses photouncaging to bypass diffusion problems of external bolus dosing and it further uses Halo protein tethering to limit the concentration of electrophile building up and potentially limit locale release. Based on clearance mechanisms for HNE as a well-studied RES, we have estimated that the maximal amount of HNE accrued in cells at a given time by this method is $1 \mu \mathrm{M}$, and later measured total HNE released intracellularly to be around $5 \mu \mathrm{M}$, consistent with the fact that the pulse of electrophile released lasts for around 5 minutes. For this reason, the amount of HNE is elevated marginally, by an ephemeral burst of a biorthogonal electrophile that can be detected with high precision at low occupancy on a protein of interest. In this way, fractional occupancy of the exogenous electrophile is low (akin to pseudo first order conditions dominated by true second-order rate constants), and the perturbation of the system due to electrophile exposure is also minimal. Using this strategy, we have argued that "the best" sensors are likely to be found. One of the sensors we identified using this strategy was Ube2V2. This protein was not known at the time to be an electrophile sensor, but subsequent experiments showed that it was able to sense low micromolar concentrations of electrophiles over a period of a few minutes.

G-REX requires photocaging of the electrophile and also ectopic expression of Halo or a similar protein. It remains to be fully understood how Halo expression affects cells and organisms, although we have 
shown that a number of model organisms can be engineered to express functional Halo proteins. Another critical issue is that the molecules used must have a bioorthogonal handle for functionalization (e.g., alkyne). As we discussed above, for molecules like (dimethyl)fumarate, this approach is not well established. Radiolabeling is one potential remedy to this issue. These are drawbacks and limitations, but similar methods have been used in a wide range of model organisms and the data can be further investigated in vitro, using mutagenesis, or fed into pipelines we describe below.

The quantitation problem

Chemical probes should be applied quantitatively de rigueur. Indeed, when studying a single protein, such measurements are a ratio of input over output. This sort of measurement is important because proposed mechanisms must account for occupancy. For instance, if a labeling event is proposed to inhibit the enzyme, $1 \%$ occupancy is unlikely to be sufficient, unless a very specific mechanism is at play. Similar applies to stimulation events: $2 \%$ occupation by an oxidation event doubling protein activity leads to negligible change in overall activity (4\%). The example of ABPP exemplifies the issues with not measuring labeling directly: the pleotropicity of electrophile and oxidant dosing/upregulation systems, and the fact that allosteric regulation of sensors is far from uncommon, means occupancy of a specific entity cannot be calculated.

\section{The fold change problem}

Obviously, when assessing "proteome-wide" labeling, such absolute quantitation of occupancy is realistically not possible, and perhaps not even necessary. Thus, fold change relative to a "control" is used as a default. However, this transformation is not without issue. It is possible that proteins that are significantly modified in the ground state, or have reached a fractional load of modification that is close to maximum in the ground state may be undervalued or considered unreactive. In the case of oxidation, it is now believed that many proteins are not directly modified by peroxide, but through change of hands. Such proteins may not even show elevated modification upon bolus dosing of oxidants, but may have an elevated basal oxidation level, or latency. Indeed, proteins that are very unmodified in the basal 
state are more likely to be enriched as even a collision-limited interaction with an electrophile may be enough to give a significant change, especially if clearance mechanisms are (locally) compromised. For these reasons, it is clear that it is hard to determine what a meaningful fold change be, and indeed that that interpretation could be context-dependent. Although this is clearly arbitrary, it would appear that a minimal fold change of 2 , and experimentation with replicates, at least 3 should be minimally considered, and individual standard deviation be shown.

\section{Phenotypes}

Biology is essentially a study of signaling and phenotypes. Thus, a complementary way to investigate reactive molecule signaling is to try to create a specific modification event, and investigate phenotypic behaviors directly attributable to it. Showing that a certain percentage labeling by a specific electrophile can elicit a specific phenotype change, can then be used to triage if a modification be impactful on a protein's behavior in a cell, or can be cross-correlated against probe-based profiling methods to see if the numbers match. When this laboratory started, we proposed that we could fashion a specific modified state of a protein in a cell, in a traceless manner, and in that way we could assay the function of the specific modification in an otherwise perturbed cell. By virtue of the experiment, which we will describe later, the precise chemotype labeled on the target can be controlled, and as the method is non-toxic downstream signaling can also be readout in an otherwise unperturbed cell many hours post the "stressor" event. Such an approach sidesteps many of the problems above, and further brings a level of quantitation and accuracy that many of the above methods do not have. This method is called T-REX (Fig. 12B-C).

T-REX uses a fusion of a protein of interest to Halo protein to allow localization of a photocaged electrophile in the vicinity of the protein of interest. Once cells expressing this halo-protein of interest fusion have been treated with a photocaged precursors to the electrophile of interest that can bind irreversibly to Halo, and the excess ligand has been washed away, the photocage and protein of interest are localized in cells in a 1:1 stoichiometry. Upon photouncaging, should the protein of interest be 
reactive with the electrophile of interest, irreversible labeling occurs at a specific nucleophilic residue, giving an electrophile labelled state that can be identified by mass spectrometry, or using biorthogonal chemistry. As the signal corresponding to $100 \%$ is defined as the signal observed on the Halo protein prior to photouncaging, and the signal delivered to the protein of interest is also expressible as a fraction of that value, absolute occupancy obtained by each procedure is calculable.

Using T-REX a good number of sensors has been shown to be functionally reactive in cells, in a variety of model organisms. Critically, many of these signaling molecules function at low ligand occupancy on their target proteins. One interesting example of data derived from T-REX is the divergence in reactivity of Akt isoforms with 4-hydroxynonenal. It transpires that Akt3 is a protein that is modified by electrophiles significantly when 4-hydroxynonenal is released under T-REX. This process leads to significant inhibition of Akt activity in cells, and in zebrafish. Akt2 is less reactive to electrophiles than Akt3, but does undergo labeling under T-REX. But this event is not sufficient to trigger downstream signaling, i.e loss in Akt activity in cells or fish. Akt1 is not electrophile sensitive at all. Akt3 senses electrophiles through a unique cysteine, C119, that exists in a hypervariable loop region, which explains why Akt3 is the most electrophile sensitive protein out of the 3 isoforms of Akt.

Taking these data together, we proposed that we could derive selective Akt3 inhibitors, from Akttargeting non-covalent inhibitors. By attaching an HNE-like appendage to a molecule that inhibits all 3 isoforms of Akt, that is now in phase II clinical trials, we showed that this inhibitor was a selective inhibitor of Akt3. This inhibition arose by virtue of better inhibition kinetics of covalent labeling for Akt3, versus Akt2 (Akt1 was unreactive), and also because the inhibition exerted its influence over Akt3 in a dominant negative fashion, whereas there was no such effect on Akt2. The same dominant negative inhibition effect was found for the parent electrophile, HNE, inhibiting Akt3 versus Akt2. Thus, we showed that our inhibitors mimicked the way the natural electrophile inhibited with the protein.

In some respects, T-REX can be considered as an extension of earlier methods, such as CALI, which localized (a) reactive biological oxidant(s) to a specific target protein due to a photo-stimulus, leading 
to its inactivation or destruction. However, these methods were limited in several ways. One these were only able to inactivate their targets and little if any work was put in to stimulate proteins in this manner. Secondly, these techniques are often unable to generate a specific chemotype. And perhaps most importantly, these methods are not stoichiometry limited, but rather serve as overload methods. Thus release of the battery of oxidants during photocaging is hugely dependent on incident light, concentration of oxygen, and critically timing of the experiment. Furthermore, as oxidation creates reversible linkages, rendering the stoichiometry of modification is overall hard to assess, especially if it were to occur with low occupancy. Thus, these systems emerged to be great for (non-physiological) ablation, but overall poor for studying signaling. It should be noted that with improved understanding of biological ROS generators, and the controls we have developed, it is possible that this system bears reinvestigation ${ }^{61}$.

T-REX has several intrinsic issues, which we have discussed at length. The most common issue that is currently levied is whether T-REX reflects the proclivity for a protein to be modified in an "endogenous" setting. We have shown that hits from T-REX show uniquely rapid labeling by HNE in vitro and we have also shown that hits from G-REX, a technique that picks out the best sensors based on collision-limited labeling, are also effectively labeled by T-REX. On the other hand, many proteins are not efficiently labeled by T-REX and several proteins that are reported to be HNE sensors do not undergo efficient labeling in vitro. Thus T-REX appears to be a fair and controlled test of electrophile sensitivity that can be carried out in cells, and model organisms. 
A

\section{G-REX}

Cells grow in medium with heavy isotopes

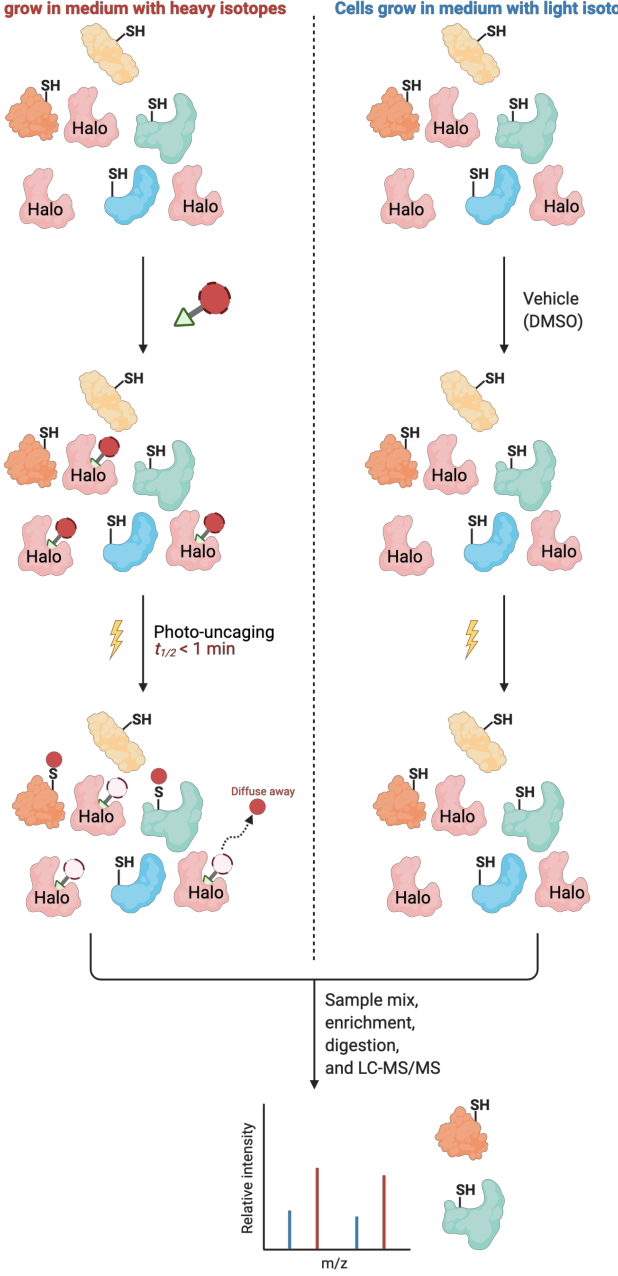

C

\section{$\underline{T-R E X}$}
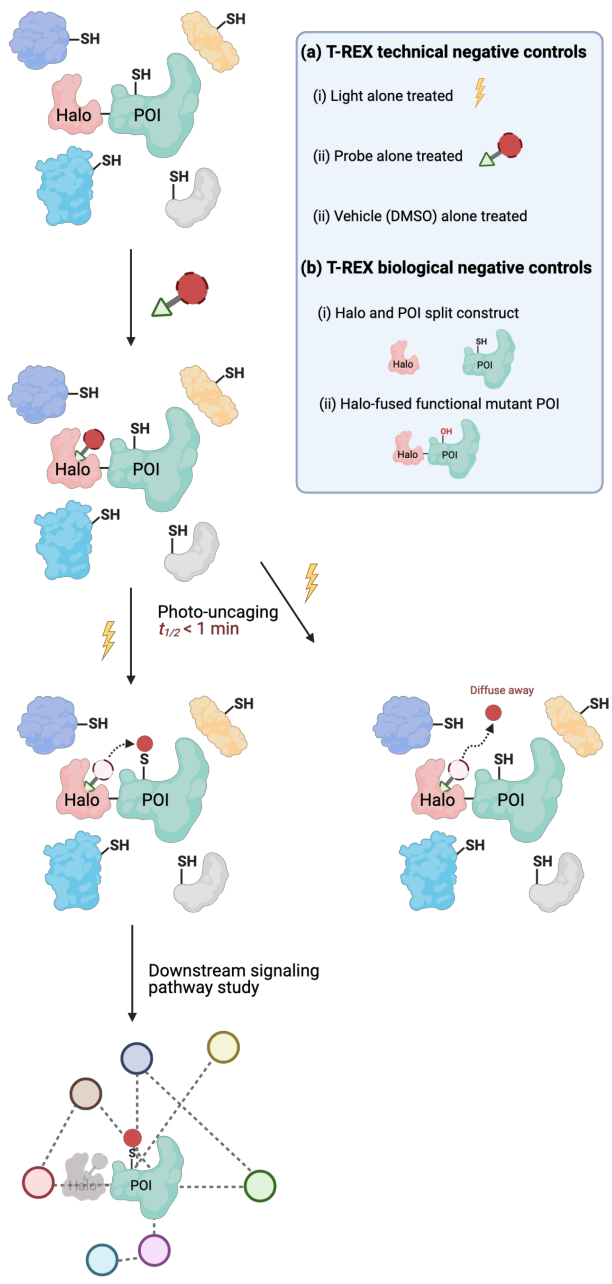

B

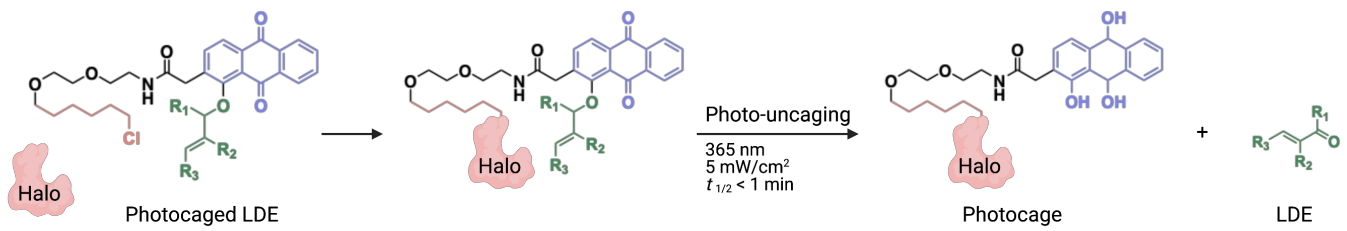

Figure 12. (A) G-REX. Cells or animals expressing HaloTag (a protein that reacts specifically, and irreversibly with chloroalkane-bearing small-molecule ligands) are cultured in media with different isotopically labeled amino acids (i.e., SILAC-labeled cells). Experiment groups (left) are treated with photocaged LDE of interest (bearing a chloroalkane to allow 1:1 binding of the probe to Halo), while the other group is treated with vehicle, e.g., DMSO. Light is exposed to both samples. In the probe-treated set, proteins within the proximity of Halo that are kinetically privileged to react with the limited amount of released LDE (1-5 $\mu M)$ are modified. Samples from each group are then mixed equally, enriched, digested, and subjected to LC-MS/MS to rank each native protein's sensitivity to LDE of interest quantitatively. (B) T-REX. Cells or animals expressing HaloTag-fused protein of interest (POI) are treated with photocaged LDE (bearing a chloroalkane to allow 1:1 binding of the probe to Halo). Upon light exposure, LDE is released in the vicinity of POI. If the POI can sense the LDE, it will be covalently modified (left). 
The covalent modification could trigger/regulate the downstream signaling events of the POI, which is useful for interrogating signaling events post LDE modifications. However, if the POI is not a sensor of LDE, the released LDE will simply diffuse away. Inset: A suite of negative controls established for T-REX: (a) technical T-REX controls, comprising (i) light alone treated control; (ii) probe alone treated control; and (iii) DMSO alone treated control; and (b) biological T-REX controls wherein identical experiment as in T-REX is performed except that either: (i) Halo and POI are expressed as two separate proteins as opposed to fusion construct; or (ii) LDE-sensing-defective but otherwise-functional mutant POI (e.g., Ala or Ser) is expressed as Halo fusion construct. In both cases, POI is not labeled by electrophile post T-REX and should the T-REX experimental data using Halo-POI fusion construct be valid, then this control experiment with non-fused construct should result in elimination of the signaling output measured otherwise under T-REX. (C) Photocage LDE probe. The probe can covalently bind to HaloTag by hexyl chloride moiety (orange). Upon light exposure, LDE (green) is released from anthraquinone-based photocage (purple). Photo-uncaging condition: $365 \mathrm{~nm}, 5 \mathrm{~mW} / \mathrm{cm}^{2}, t_{1 / 2}<1 \mathrm{~min}$.

\section{Epilogue}

Reactive molecule signaling is deceptively simple: it is just a bimolecular reaction. It is thus unsurprising that biochemists, chemists and chemical biologists have been drawn to study this process. However, when played out the in backdrop of thousands of proteins, hundreds of thousands of nucleophiles, myriad locales, and a plethora of potential interacting partners, as we know from our everyday lives, things can get complicated. It is how these differences have occurred and what those differences convey to the "chosen" reactive partners that makes this field fascinating. In this way chemistry has to give way to biological investigations interrogating specific modification processes. Thus, over the years redox signaling has been studied, we have thus transitioned from in vitro experimentation to cell-based investigations. We are moving to a more protein-centric understanding defined by precision experimentation using probes and chemical biological techniques that are applicable in cells and organisms. This shift is far from over, and overall our understanding, just like the techniques deployed in this field, is still in their infancy. So, this is a great time for new players to enter the arena, and it is a wonderful backdrop in which to learn and apply multidisciplinary concepts.

Author contributions: concept and writing: M.J.C.L., Y.A.; figures: K-T.H. All authors agree to the final version of the manuscript. Funding: EPFL, SNSF project funding 184729. 
References

1 Parvez, S., Long, M. J. C., Poganik, J. R. \& Aye, Y. Redox signaling by reactive electrophiles and oxidants. Chem Rev 118, 8798-8888, (2018).

2 Brown, H. C., Brewster, J. H. \& Shechter, H. An Interpretation of the Chemical Behavior of Fiveand Six-membered Ring Compounds1. Journal of the American Chemical Society 76, 467-474, (1954).

3 Poganik, J. R. \& Aye, Y. Electrophile signaling and emerging immuno- and neuro-modulatory electrophilic pharmaceuticals. Frontiers in Aging Neuroscience 12, doi.org/10.3389/fnagi.2020.00001, (2020).

4 Cuadrado, A., Rojo, A. I., Wells, G., Hayes, J. D., Cousin, S. P., Rumsey, W. L., Attucks, O. C., Franklin, S., Levonen, A.-L., Kensler, T. W. \& Dinkova-Kostova, A. T. Therapeutic targeting of the NRF2 and KEAP1 partnership in chronic diseases. Nature Reviews Drug Discovery, In press, DOI: 10.1038/s41573-41018-40008-x, (2019).

5 Hansen, R. E., Roth, D. \& Winther, J. R. Quantifying the global cellular thiol-disulfide status. Proceedings of the National Academy of Sciences 106, 422-427, (2009).

6 Antelmann, H. \& Helmann, J. D. Thiol-based redox switches and gene regulation. Antioxid Redox Signal 14, 1049-1063, (2011).

7 Marinho, H. S., Real, C., Cyrne, L., Soares, H. \& Antunes, F. Hydrogen peroxide sensing, signaling and regulation of transcription factors. Redox Bio/ 2, 535-562, (2014).

8 Peskin, A. V. \& Winterbourn, C. C. Kinetics of the reactions of hypochlorous acid and amino acid chloramines with thiols, methionine, and ascorbate. Free Radical Biology and Medicine 30, 572-579, (2001).

9 Reich, H. J. \& Hondal, R. J. Why Nature Chose Selenium. ACS Chemical Biology 11, 821-841, (2016).

10 Toledano, M. B., Fourquet, S. \& D’Autréaux, B. in Signal Transduction: Pathways, Mechanisms and Diseases (ed Ari Sitaramayya) 313-336 (Springer Berlin Heidelberg, 2010).

11 Pattison, D. I. \& Davies, M. J. Kinetic Analysis of the Reactions of Hypobromous Acid with Protein Components: Implications for Cellular Damage and Use of 3-Bromotyrosine as a Marker of Oxidative Stress. Biochemistry 43, 4799-4809, (2004).

12 Richardson, D. E., Regino, C. A. S., Yao, H. \& Johnson, J. V. Methionine oxidation by peroxymonocarbonate, a reactive oxygen species formed from $\mathrm{CO} /$ bicarbonate and hydrogen peroxide. Free Radic Biol Med 35, 1538-1550, (2003).

13 Kim, J. \& Huang, C.-H. Reactivity of Peracetic Acid with Organic Compounds: A Critical Review. ACS ES\&T Water 1, 15-33, (2021).

14 Du, P., Liu, W., Cao, H., Zhao, H. \& Huang, C.-H. Oxidation of amino acids by peracetic acid: Reaction kinetics, pathways and theoretical calculations. Water Research X1, 100002, (2018).

15 Sivey, J. D., Howell, S. C., Bean, D. J., McCurry, D. L., Mitch, W. A. \& Wilson, C. J. Role of lysine during protein modification by $\mathrm{HOCl}$ and $\mathrm{HOBr}$ : halogen-transfer agent or sacrificial antioxidant? Biochemistry 52, 1260-1271, (2013).

16 Hawkins, C. L., Pattison, D. I. \& Davies, M. J. Hypochlorite-induced oxidation of amino acids, peptides and proteins. Amino Acids 25, 259-274, (2003).

17 Asada, K. \& Kanematsu, S. Reactivity of Thiols with Superoxide Radicals. Agricultural and Biological Chemistry 40, 1891-1892, (2014).

18 Kobayashi, K., Hayashi, K. \& Sono, M. Effects of tryptophan and pH on the kinetics of superoxide radical binding to indoleamine 2,3-dioxygenase studied by pulse radiolysis. J Biol Chem 264, 15280-15283, (1989).

19 Taylor, A. A. Side by side: The work of Elizabeth and James Miller. J Biol Chem 292, 1200812009, (2017). 
20 Lin, D., Lee, H. G., Liu, Q., Perry, G., Smith, M. A. \& Sayre, L. M. 4-Oxo-2-nonenal is both more neurotoxic and more protein reactive than 4-hydroxy-2-nonenal. Chem Res Toxicol 18, 12191231, (2005).

21 Tacka, K. A., Dabrowiak, J. C., Goodisman, J. \& Souid, A. K. Kinetic analysis of the reactions of 4-hydroperoxycyclophosphamide and acrolein with glutathione, mesna, and WR-1065. Drug Metab Dispos 30, 875-882, (2002).

22 Bent, G.-A., Maragh, P. \& Dasgupta, T. In vitro studies on the reaction rates of acrylamide with the key body-fluid thiols I-cysteine, glutathione, and captopril. Toxicology Research 3, 445446, (2014).

23 Long, M. J. C. \& Aye, Y. Privileged electrophile sensors: A resource for covalent drug development. Cell Chem Biol 24, 787-800, (2017).

24 Spiess, P. C., Deng, B., Hondal, R. J., Matthews, D. E. \& van der Vliet, A. Proteomic profiling of acrolein adducts in human lung epithelial cells. J Proteomics 74, 2380-2394, (2011).

25 Reddy, H., Duffy, A., Holtzman, N. G. \& Emadi, A. The role of $\beta$-elimination for the clinical activity of hypomethylating agents and cyclophosphamide analogues. Am J Cancer Ther Pharmacol 3, 1-8, (2016).

26 Rémion, J., Dumont, W. \& Krief, A. New regiospecific routes to olefins from $\beta$-hydroxy selenides: - a stereospecific deoxygenation of epoxides - a [C,C] connective route to olefins. Tetrahedron Letters 17, 1385-1388, (1976).

27 Shaw, E. \& Ruscica, J. The reactivity of his-57 in chymotrypsin to alkylation. Archives of Biochemistry and Biophysics 145, 484-489, (1971).

28 Jackson, P. A., Widen, J. C., Harki, D. A. \& Brummond, K. M. Covalent Modifiers: A Chemical Perspective on the Reactivity of $\alpha, \beta$-Unsaturated Carbonyls with Thiols via Hetero-Michael Addition Reactions. J Med Chem 60, 839-885, (2017).

29 Mclemore, R., Robb, S. A., Lee, B. H., Caplan, M. R. \& Vernon, B. L. Michael-type addition reactions in NIPAAm-cysteamine copolymers follow second order rate laws with steric hindrance. Ann Biomed Eng 37, 2416-2425, (2009).

30 Friguet, B., Szweda, L. I. \& Stadtman, E. R. Susceptibility of glucose-6-phosphate dehydrogenase modified by 4-hydroxy-2-nonenal and metal-catalyzed oxidation to proteolysis by the multicatalytic protease. Arch Biochem Biophys 311, 168-173, (1994).

31 Kisselev, A. F., Akopian, T. N., Castillo, V. \& Goldberg, A. L. Proteasome Active Sites Allosterically Regulate Each Other, Suggesting a Cyclical Bite-Chew Mechanism for Protein Breakdown. Molecular Cell 4, 395-402, (1999).

32 Shringarpure, R., Grune, T., Sitte, N. \& Davies, K. J. A. 4-Hydroxynonenal-modified amyloid- $\beta$ peptide inhibits the proteasome: possible importance in Alzheimer's disease. Cellular and Molecular Life Sciences CMLS 57, 1802-1809, (2000).

33 Friguet, B. \& Szweda, L. I. Inhibition of the multicatalytic proteinase (proteasome) by 4hydroxy-2-nonenal cross-linked protein. FEBS Letters 405, 21-25, (1997).

34 Rhee, S. G. Overview on Peroxiredoxin. Mol Cells 39, 1-5, (2016).

35 Jung, T., Engels, M., Kaiser, B. \& Grune, T. Distribution of oxidized and HNE-modified proteins in U87 cells. Biofactors 24, 165-170, (2005).

36 Petkovic, I., Bresgen, N., Gilardoni, E., Regazzoni, L., Uchida, K., Aldini, G., Siems, W. \& Eckl, P. In Vitro Aging of Human Skin Fibroblasts: Age-Dependent Changes in 4-Hydroxynonenal Metabolism. Antioxidants (Basel) 9, (2020).

37 Musicco, C., Capelli, V., Pesce, V., Timperio, A. M., Calvani, M., Mosconi, L., Zolla, L., Cantatore, P. \& Gadaleta, M. N. Accumulation of overoxidized Peroxiredoxin III in aged rat liver mitochondria. Biochimica et Biophysica Acta (BBA) - Bioenergetics 1787, 890-896, (2009).

38 Wabnitz, G. H., Goursot, C., Jahraus, B., Kirchgessner, H., Hellwig, A., Klemke, M., Konstandin, M. H. \& Samstag, Y. Mitochondrial translocation of oxidized cofilin induces caspaseindependent necrotic-like programmed cell death of T cells. Cell Death \& Disease 1, e58-e58, (2010). 
39 Long, M. J. C., Wang, L. \& Aye, Y. Getting the Right Grip? How Understanding Electrophile Selectivity Profiles Could Illuminate Our Understanding of Redox Signaling. Antioxid Redox Signal (doi: 10.1089/ars.2019.7894), (2019).

40 Zaro, B. W., Whitby, L. R., Lum, K. M. \& Cravatt, B. F. Metabolically Labile Fumarate Esters Impart Kinetic Selectivity to Irreversible Inhibitors. Journal of the American Chemical Society 138, 15841-15844, (2016).

41 Lyublinskaya, O. \& Antunes, F. Measuring intracellular concentration of hydrogen peroxide with the use of genetically encoded H2O2 biosensor HyPer. Redox Biology 24, 101200, (2019).

42 Forman, H. J., Bernardo, A. \& Davies, K. J. A. What is the concentration of hydrogen peroxide in blood and plasma? Archives of Biochemistry and Biophysics 603, 48-53, (2016).

43 Zabłocka-Słowińska, K., Płaczkowska, S., Skórska, K., Prescha, A., Pawełczyk, K., Porębska, I., Kosacka, M. \& Grajeta, H. Oxidative stress in lung cancer patients is associated with altered serum markers of lipid metabolism. PLoS One 14, e0215246, (2019).

44 Zhu, H., Tamura, T., Fujisawa, A., Nishikawa, Y., Cheng, R., Takato, M. \& Hamachi, I. Imaging and Profiling of Proteins under Oxidative Conditions in Cells and Tissues by HydrogenPeroxide-Responsive Labeling. J Am Chem Soc 142, 15711-15721, (2020).

45 Stephan, J. R., Yu, F., Costello, R. M., Bleier, B. S. \& Nolan, E. M. Oxidative Post-translational Modifications Accelerate Proteolytic Degradation of Calprotectin. J Am Chem Soc 140, 1744417455, (2018).

46 Liu, H., Ponniah, G., Neill, A., Patel, R. \& Andrien, B. Accurate determination of protein methionine oxidation by stable isotope labeling and LC-MS analysis. Anal Chem 85, 1170511709, (2013).

47 Vajrychova, M., Salovska, B., Pimkova, K., Fabrik, I., Tambor, V., Kondelova, A., Bartek, J. \& Hodny, Z. Quantification of cellular protein and redox imbalance using SILAC-iodoTMT methodology. Redox Biol 24, 101227, (2019).

48 Petroski, M. D. \& Deshaies, R. J. Redundant degrons ensure the rapid destruction of Sic1 at the G1/S transition of the budding yeast cell cycle. Cell Cycle 2, 410-411, (2003).

49 Adusumalli, S. R., Rawale, D. G. \& Rai, V. Aldehydes can switch the chemoselectivity of electrophiles in protein labeling. Organic \& Biomolecular Chemistry 16, 9377-9381, (2018).

50 Benitez, L. V. \& Allison, W. S. The inactivation of the acyl phosphatase activity catalyzed by the sulfenic acid form of glyceraldehyde 3-phosphate dehydrogenase by dimedone and olefins. $J$ Biol Chem 249, 6234-6243, (1974).

51 Klomsiri, C., Nelson, K. J., Bechtold, E., Soito, L., Johnson, L. C., Lowther, W. T., Ryu, S. E., King, S. B., Furdui, C. M. \& Poole, L. B. Use of dimedone-based chemical probes for sulfenic acid detection evaluation of conditions affecting probe incorporation into redox-sensitive proteins. Methods Enzymol 473, 77-94, (2010).

52 Huang, J., Willems, P., Wei, B., Tian, C., Ferreira, R. B., Bodra, N., Martínez Gache, S. A., Wahni, K., Liu, K., Vertommen, D., Gevaert, K., Carroll, K. S., Van Montagu, M., Yang, J., Van Breusegem, F. \& Messens, J. Mining for protein S-sulfenylation in Arabidopsis uncovers redoxsensitive sites. Proc Natl Acad Sci U S A 116, 21256-21261, (2019).

53 Yang, M., Li, W., Harberg, C., Chen, W., Yue, H., Ferreira, R. B., Wynia-Smith, S. L., Carroll, K. S., Zielonka, J., Flaumenhaft, R., Silverstein, R. L. \& Smith, B. C. Cysteine sulfenylation by CD36 signaling promotes arterial thrombosis in dyslipidemia. Blood Advances 4, 4494-4507, (2020).

54 Liu, J., Zhang, Z. \& Rozovsky, S. Selenoprotein K form an intermolecular diselenide bond with unusually high redox potential. FEBS Lett 588, 3311-3321, (2014).

55 Yang, J., Gupta, V., Carroll, K. S. \& Liebler, D. C. Site-specific mapping and quantification of protein S-sulphenylation in cells. Nature Communications 5, 4776, (2014).

56 Jencks, W. P. in Nucleophilicity Vol. 215 Advances in Chemistry Ch. 10, 155-167 (American Chemical Society, 1987). 
57 Peroni, E., Scali, V., Balestri, F., Cappiello, M., Mura, U., Del Corso, A. \& Moschini, R. Pathways of 4-Hydroxy-2-Nonenal Detoxification in a Human Astrocytoma Cell Line. Antioxidants (Basel) 9, (2020).

58 Škulj, S., Vazdar, K., Margetić, D. \& Vazdar, M. Revisited Mechanism of Reaction between a Model Lysine Amino Acid Side Chain and 4-Hydroxynonenal in Different Solvent Environments. The Journal of Organic Chemistry 84, 526-535, (2019).

59 Kuljanin, M., Mitchell, D. C., Schweppe, D. K., Gikandi, A. S., Nusinow, D. P., Bulloch, N. J., Vinogradova, E. V., Wilson, D. L., Kool, E. T., Mancias, J. D., Cravatt, B. F. \& Gygi, S. P. Reimagining high-throughput profiling of reactive cysteines for cell-based screening of large electrophile libraries. Nat Biotechnol DOI: 10.1038/s41587-020-00778-3, (2021).

60 Chen, Y., Liu, Y., Lan, T., Qin, W., Zhu, Y., Qin, K., Gao, J., Wang, H., Hou, X., Chen, N., Friedmann Angeli, J. P., Conrad, M. \& Wang, C. Quantitative Profiling of Protein Carbonylations in Ferroptosis by an Aniline-Derived Probe. Journal of the American Chemical Society 140, 47124720, (2018).

61 Westberg, M., Etzerodt, M. \& Ogilby, P. R. Rational design of genetically encoded singlet oxygen photosensitizing proteins. Current Opinion in Structural Biology 57, 56-62, (2019). 